\title{
Skeletonized Wave-Equation Refraction Inversion With Autoencoded Waveforms
}

\author{
$\mathrm{Han} \mathrm{Yu}^{\oplus}$, Yuqing Chen, Sherif M. Hanafy, and Gerard T. Schuster
}

\begin{abstract}
We present a method that skeletonizes the first arriving seismic refractions by machine learning and inverts them for the subsurface velocity model. In this study, first arrivals can be compressed in a low-rank sense with their skeletal features extracted by a well-trained autoencoder. Empirical experiments suggest that the autoencoder's $1 \times 1$ or $2 \times 1$ latent vectors vary continuously with respect to the input seismic data. It is, therefore, reasonable to introduce a misfit functional measuring the discrepancies between the predicted and the observed data in a low-dimensional latent space. The benefit of this approach is that an elaborated autoencoding neural network not only refines intrinsic information hidden in the refractions but also improves the quality of inversion for a reliable background velocity model. Numerical tests on both synthetic and field data demonstrate the effectiveness of this method, especially in recovering the low-to-intermediate wavenumber parts of the subsurface velocity distribution. Comparisons are made with the other three relevant methods, the wave-equation travel-time (WT) inversion, the envelope inversion, and the full waveform inversion (FWI). As expected, the cycle skipping problem is alleviated due to the reduction of dimensions of data space. This method outperforms the envelope inversion in resolution, and it is no worse than WT. Moreover, there is no need for careful manual travel-time picking with this methodology. In general, this inversion framework provides an extendable strategy to compress any input data for reconstructing high-dimensional physical parameters.
\end{abstract}

Index Terms-Autoencoder, refractions, skeletonization, waveform inversion.

\section{INTRODUCTION}

$\mathbf{F}$ ULL waveform inversion (FWI) has been proven to be an effective method for high-resolution imaging the Earth [1] by iteratively refining velocity models with reverse time

Manuscript received March 1, 2020; revised August 11, 2020 and October 19, 2020; accepted December 13, 2020. This work was supported in part by the King Abdullah University of Science and Technology (KAUST); in part by the Nanjing University of Posts and Telecommunications (NUPT); in part by the China Postdoctoral Science Foundation under Grant 2018T110531; and in part by the National Natural Science Foundation of China under Grant 11501302, Grant 61972209, and Grant 61672016. (Corresponding author: Han Yu.)

Han Yu is with the School of Computer Science, Nanjing University of Posts and Telecommunications, Nanjing 210023, China, also with the Jiangsu Key Laboratory of Big Data Security and Intelligent Processing, Nanjing University of Posts and Telecommunications, Nanjing 210023, China, and also with Suzhou Keda Technology Company Ltd., Suzhou 215011, China (e-mail: han.yu@njupt.edu.cn).

Yuqing Chen and Gerard T. Schuster are with the Division of Physical Science and Engineering, King Abdullah University of Science and Technology, Thuwal 23955-6900, Saudi Arabia.

Sherif M. Hanafy is with the College of Petroleum Engineering and Geosciences, King Fahd University of Petroleum and Minerals, Dhahran 31261, Saudi Arabia.

Color versions of one or more figures in this article are available at https://doi.org/10.1109/TGRS.2020.3046093.

Digital Object Identifier 10.1109/TGRS.2020.3046093 migration (RTM) of the residuals [2], [3]. However, almost all successful inversions rely on accurate initial velocity models [4], where most misfit functionals of FWI can be easily trapped into local minimum due to the famous cycleskipping issue [5]. Remedies to this problem tend to utilize the information hidden in the low-frequency signals of the recorded seismic data. One of the most successful methods is the multiscale approach [6] that gradually inverts for the velocity models with low-to-high wavenumbers [7]. Not long ago, the suboffset and time-lag axes were used for migration velocity analysis (MVA) to accelerate the overall convergence of FWI in the tomographic FWI [8]. Later, a data-adaptive matching strategy was proposed in [9] to partially overcome the cycle-skipping problem. Other methods, such as the wavefield reconstruction inversion, were also proposed in [10] to avoid the necessities of kinematically accurate initial models, and it might rely on building a proper misfit function with regularization.

$\mathrm{Wu}$ and Chen [11] employed more information from the envelope of seismic data to invert for the salt body, which automatically incorporated more ultralow frequency signals so that the misfit functionals are less sensitive to cycle-skipping. Kwon et al. [12] have made a series of advances in the Laplace-Fourier domain to invert the data with low frequencies, therefore making their inversions more robust than conventional FWI. Recently, Yao et al. [13] and Chen et al. [14] revised the velocity models gradually from near-offset to faroffset reflections so that the objective functions can more likely reach the global minima and the corrected velocity models can approximate the true model. To focus on matching the kinematic information rather than the dynamics, Sun and Schuster [15] and Fu et al. [16] developed a phase mismatch objective function that is largely insensitive to the amplitude mismatch between the predicted and observed arrivals.

The nonlinearity of the FWI function usually prevents the updated velocity model from converging to the correct one. To overcome this problem and the high-frequency approximation of ray-based tomography, Luo et al. [17] and Luo and Schuster [18] inverted travel time using the wave-equation. Inverting refraction travel times with the wave equation not only reduces the complexity of the misfit functional [19] but also accelerates the convergence of iterative inversion. Nevertheless, only inverting the travel times of first arrivals limits the tomogram to be a low resolved approximation to the subsurface velocity model.

Theoretically, the key idea of skeletonized inversion is to invert skeletal features in the data that are strongly dependent on the model parameters of interest. For example, changes 


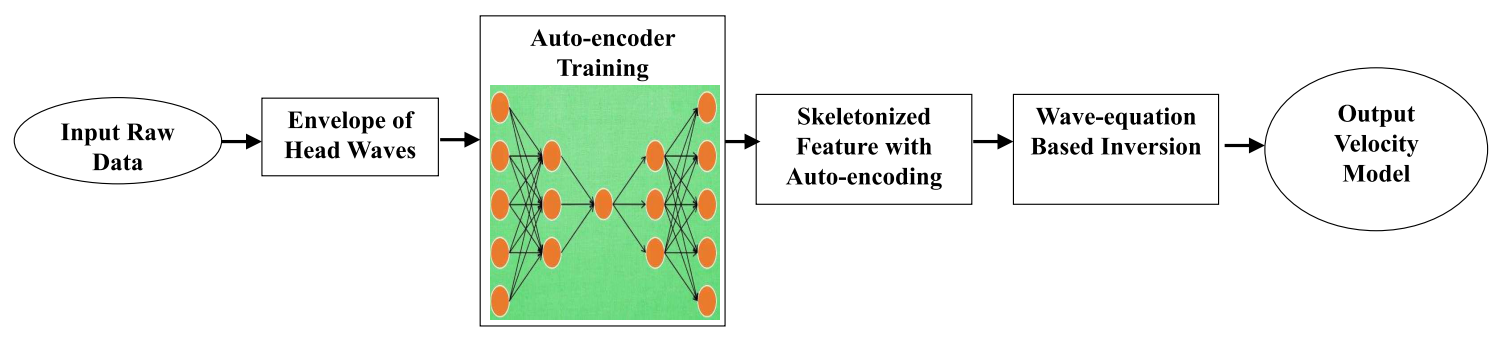

Fig. 1. Framework for skeletonized wave-equation inversion of AE head waves.

in the velocity model mostly influence the travel times of reflections and diving waves, not their amplitudes. Another example is by Dutta [20] who inverted the shift in peak frequencies of arrivals for the $Q$ distribution and Li et al. [21] who inverted the dispersion curves of surface waves for the shear-velocity distribution. Lu et al. [22] summarized these methods to describe the development of them and their future applications.

In all the abovementioned skeletonized inversion methods, once the data set is skeletonized, the misfit function measures the difference between the predicted and the observed seismograms in the compressed data domain. The adjoint sources are weighted by the skeletonized residuals and then backprojected into the model to update the target parameters.

To generalize the skeletonized inversion procedure to any type of skeletal data, Chen and Schuster [23] developed the Newtonian machine learning (NML) method. Here, they used the compressed latent space variables of an autoencoder [24]-[26] as the skeletonized data. They then used the implicit function theorem to invert these latent variables for the model parameters of interest. In their formula, the raw direct arrivals in a cross-well experiment are used to invert the latent variables for the velocity model, and the dimension of their proposed latent space is only one, which may undermine the quality of data restoration and inversion. Since the processed first arriving waveforms are usually less complicated, they are more suitable for the input of an autoencoder than later arrivals [27], [28].

It is, thus, proposed in this study to construct the misfit functional as the sum of squared differences between the calculated and the observed latent-space variables associated with a land seismic survey. Here, we refer to NML as autoencoded (AE) inversion. The synthetic and field data are windowed about the first arrivals, and some 1 (or 2$) \times 1$ vectors of latent-space coefficients are used for skeletonizing traces, not only the 1-D latent space vector in [23]. Therefore, all the formulas in the theoretical section are redeveloped in this study. Comprehensive results with both synthetic and field data show the efficiency of this approach, where the major benefit is that refractional seismic features automatically skeletonized by an autoencoding network can naturally participate in the inversion based on the wave equation. Also, the travel times do not need to be precisely picked for the first arrivals.

This article is organized into four parts. After the introduction, we explain the theory of an autoencoding neural network and how it is used for skeletonized waveform inversion. This includes the use of the implicit function theorem to derive the wave-equation formulas for the misfit gradient of the latent variables. Section III presents the numerical examples for applying this method to both a synthetic data set and a land data set. Section IV presents the conclusions and discussions.

\section{THEORY}

The skeletonized inversion scheme is divided into two parts. The first part, including Sections II-A and II-B, is to briefly introduce the autoencoding technique. The second part, including Sections II-C-II-E, shows how the implicit function theorem can be used to derive the wave-equation formulas for the misfit gradient of the latent variables.

\section{A. Autoencoder}

In this study, an autoencoding neural network serves as a tool to map the first arriving signals to the latent space first and then back to the original data space. However, the input seismic waveforms to be inverted require more simplification to reduce the complexity of training the autoencoding network [25]. Therefore, we only take the envelopes of the first arriving head waves for encoding. Once the estimated first arrivals are determined, averaging the near offset refraction wavelets within a window of 10-15 traces can help to identify the length of the time windows around the first arrivals. A Hanning window is then used to mute the later arrivals while avoiding frequency leaks. Next, we apply the Hilbert transform to the first arrivals for obtaining their envelopes. The key idea stems from the connective function in wave-equation travel time (WT), but the "travel time" here is replaced by the encoded vector (or scalar), which represents both the travel time and the normalized amplitude, that is, the envelope feature.

Fig. 1 presents the workflow of our proposed inversion method. The input raw data represent the envelopes of the refractions, as shown by the red curve in Fig. 2. There are three basic requirements for the raw data as the input of an autoencoder. First, the features of the first arriving signals should include both the travel time and the amplitude information. Second, the skeletonized features can be plugged into the inversion framework as a part of the misfit function. Third, to effectively compute the Fréchet derivatives, perturbations of the velocity model and the skeletal data can be connected by 
TABLE I

Dimensions of MAtrices For ConNecting Different LAYERS IN THE AUtoencoder

\begin{tabular}{|c|c|c|c|c|c|c|}
\hline Encoders & $W_{1}$ & $b_{1}$ & $W_{2}$ & $b_{2}$ & $W_{3}$ & $b_{3}$ \\
\hline Sizes & $\operatorname{Dim} 2 \times \operatorname{Dim} 1$ & $\operatorname{Dim} 2 \times 1$ & $\operatorname{Dim} 3 \times \operatorname{Dim} 2$ & $\operatorname{Dim} 3 \times 1$ & $2($ or 1$) \times \operatorname{Dim} 3$ & 2 (or 1$) \times 1$ \\
\hline \multicolumn{7}{|c|}{} \\
\hline Decoders & $W_{1}{ }^{\prime}$ & $b_{1}{ }^{\prime}$ & $W_{2}{ }^{\prime}$ & $b_{2}{ }^{\prime}$ & $W_{3}{ }^{\prime}$ & $b_{3^{\prime}}$ \\
\hline Sizes & $\operatorname{Dim} 1 \times \operatorname{Dim} 2$ & $\operatorname{Dim} 1 \times 1$ & $\operatorname{Dim} 2 \times \operatorname{Dim} 3$ & $\operatorname{Dim} 2 \times 1$ & $\operatorname{Dim} 3 \times 2($ or 1$)$ & $\operatorname{Dim} 3 \times 1$ \\
\hline
\end{tabular}

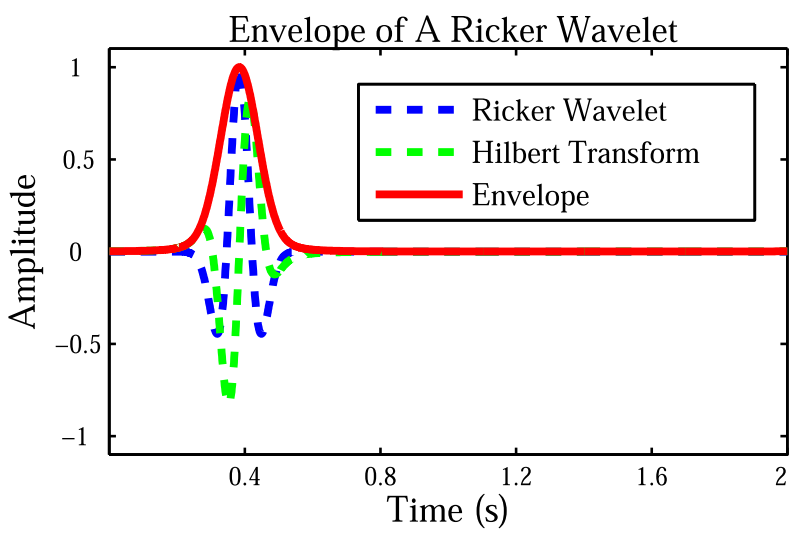

Fig. 2. Envelope of a Ricker wavelet with the frequency at peaked $30 \mathrm{~Hz}$.

a continuously differentiable function. Therefore, we choose envelopes of the first arriving refractions as the input for the autoencoding network. A prior trained autoencoding network, as a well-known unsupervised learning tool to compress data, can squeeze out the features hidden in the first arriving envelopes for the following inversion.

In theory, if the network is well-trained, the encoding and the decoding processes can be symmetric, which means that the decoder is the inverse of the encoder. With this simple assumption, we set up the network in such a way that at least their structures are alike, as shown in Fig. 3. The layers in the autoencoder are for a fully connected neural network. We expect that the decoded signals can approximate the original ones as close as possible, which means that the skeletonized features can reflect the intrinsic characteristics of the waveforms to a large extent.

\section{B. Data Training}

For the observed first arrivals of all the common shot gathers (CSGs), it is not necessary to use the entire data set for training because of the increasing cost of computation, and only a part of them can be chosen for building the autoencoder. Each seismic trace is Hilbert transformed to its envelope first and then normalized by the variance or the root mean squared energy, as shown in Fig. 2. Then, these processed envelopes $e^{(i)}$ are fed into the autoencoder network with a 1-D or 2-D latent space, where $i$ is the envelope index. Here, an increase in the latent space dimension does not lead to more computational time in training. Instead, it is expected to improve the accuracy of data compression and restoration compared with the 1-D case. An analog to this strategy is like the Fourier transform that extracts intrinsic data properties, namely the amplitude and the phase information, but only one of the two parameters cannot solely restore the original signals based on inverse FFT. However, the two mechanisms are not equivalent in which an autoencoder can be more complicated than the discrete Fourier transform. Therefore, it is worth to remark that no obvious parallels can be drawn between the two operations.

In Fig. 3, for both the encoder and the decoder subnetworks, we design three layers with symmetric dimensions. For instance, the three encoding operators with their weighting matrices' $\left(W_{1,2,3}\right)$ dimensions as $\operatorname{Dim} 2 \times \operatorname{Dim} 1, \operatorname{Dim} 3 \times \operatorname{Dim} 2$, and $1($ or 2$) \times \operatorname{Dim} 3$, where $\operatorname{Dim} 1>\operatorname{Dim} 2>\operatorname{Dim} 3$, shrink in size until the latent-space layer. If the length of every input envelope is equal to $M$, we must have $M=$ Dim1. In the latent space, there is only one vector (or scalar) as the extracted feature of one seismic trace for rebuilding the input envelope. Then, the original trace can be recovered through the inverse procedure with another three-layered network that maps the vector back to the envelope space. The dimensions of all the matrices in constructing the autoencoding neural network are presented in Table I. The minibatch gradient descending method is used to train this neural network in Pytorch.

Here, every envelope covers two or three wavelets of a trace with the number of temporal samples $M \leq 3000$. We set this threshold for $M$ by considering the computation cost of training. In fact, this threshold can be adjusted according to various applications whenever necessary. The number of input training samples into the autoencoder is just equal to the number of selected sample traces, and this number can range from thousands to millions depending on the sizes of data sets. Based on our empirical tests, three layers are adequate for extracting the important features of the data that are strongly dependent on the velocity model. Another three pairs of matrices and vectors $W_{i}^{\prime}$ and $b_{i}^{\prime}$ form the decoding procedure. To ensure autoencoding effects, Dim3, Dim2, and Dim1 should gradually approximate the length of the first arriving wavelets. The leftmost and the rightmost signals in Fig. 3 present the original envelope and the decoded envelopes. An ReLu or sigmoid function $\operatorname{Re}=\tanh ()$ is inserted into the encoding and the decoding processes. Therefore, the weighting matrices $W_{i}$ and $W_{i}^{\prime}$, and their corresponding bias vectors $b_{i}$ and $b_{i}^{\prime}(i=1,2,3)$ can be updated by training the misfit functional defined as

$$
\operatorname{ANN}\left(W_{1,2,3}, b_{1,2,3}, W^{\prime}{ }_{1,2,3}, b_{1,2,3}^{\prime}\right)=\min \sum_{i=1}^{M}\left(\hat{e}^{(i)}-e^{(i)}\right)^{2}
$$

where the decoded $\hat{e}^{(i)}$ is expressed by

$$
\begin{aligned}
\hat{e}^{(i)}= & W_{1}^{\prime} \times \operatorname{Re}\left(W_{2}^{\prime} \times \operatorname{Re}\left(W_{3}^{\prime} \times \operatorname{Re}\left(W_{3} \times \operatorname{Re}\left(W_{2}\right.\right.\right.\right. \\
& \left.\left.\left.\left.\times \operatorname{Re}\left(W_{1} \times e^{(i)}+b_{1}\right)+b_{2}\right)+b_{3}\right)+b_{3}^{\prime}\right)+b_{2}^{\prime}\right)+b_{1}^{\prime} .
\end{aligned}
$$




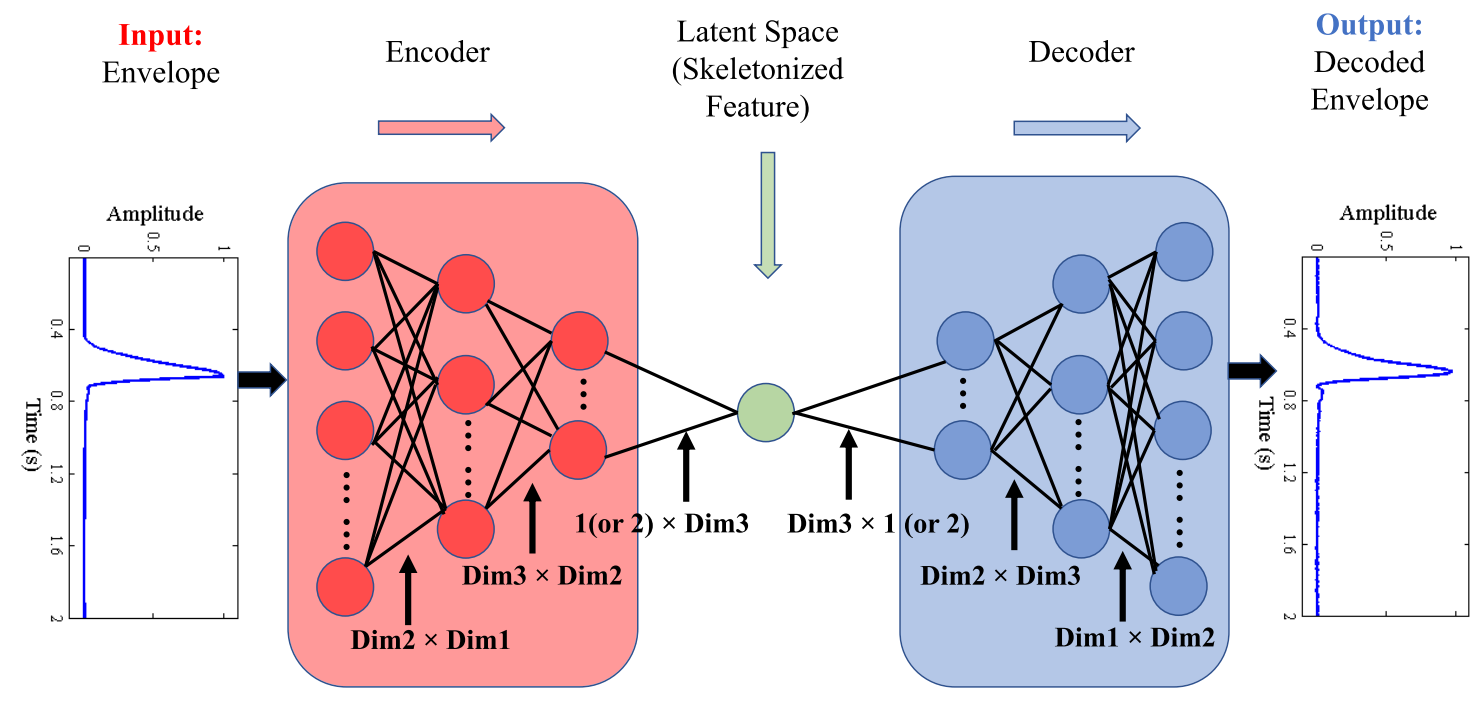

Fig. 3. Autoencoder framework for data encoding and decoding.

a)

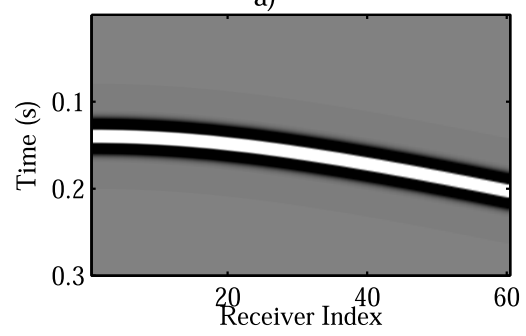

c)

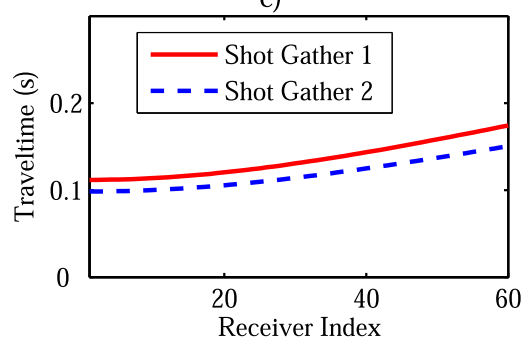

b)

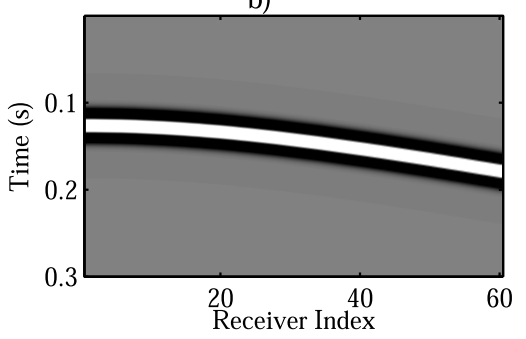

d)

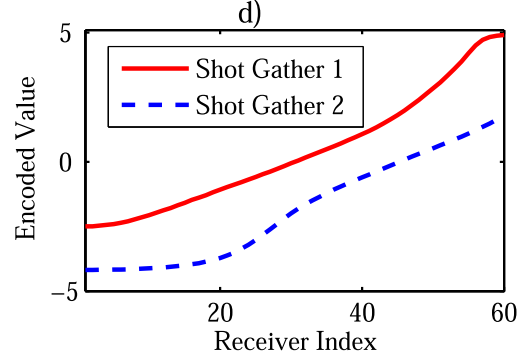

Fig. 4. Two reflecting CSGs: (a) shot gather 1 and (b) shot gather 2 with (c) their first arrival travel-time comparison and (d) encoded skeletal data comparison.

As a result, we believe that the encoded vector $\mathbf{z}=W_{3} \times$ $\operatorname{Re}\left(W_{2} \times \operatorname{Re}\left(W_{1} \times e^{(i)}+b_{1}\right)+b_{2}\right)+b_{3}$ is the best representation of the original envelope under this well-trained autoencoding neural network in the least-squares sense. This misfit function for the low-dimensional representation of the seismic data exhibits a pattern similar to that of the travel-time misfit function, as another skeletonized feature sensitive to the velocity changes. To verify this assumption, we simply build two two-layered models for testing the encoder on the reflections traveling over the media, and the only difference is the velocity distribution of their top layers: one model is with velocity $v_{\text {top }}=2.5 \mathrm{~km} / \mathrm{s}$, while the other one is with $v_{\text {top }}=3 \mathrm{~km} / \mathrm{s}$. All the other settings are identical for comparisons. Fig. 4(a) and (b) presents reflecting CSGs of two shots excited at the same location but corresponding to models with $v_{\text {top }}=2.5 \mathrm{~km} / \mathrm{s}$ and $v_{\text {top }}=3 \mathrm{~km} / \mathrm{s}$, respectively. Fig. 4(c) and (d) shows the comparisons of their travel-time curves and their encoded latent space values, respectively.
For this preliminary toy example, we train the autoencoder using half of the CSGs with $v_{\text {top }}=2.5 \mathrm{~km} / \mathrm{s}$ and half of the CSGs with $v_{\text {top }}=3 \mathrm{~km} / \mathrm{s}$, and the evidence in Fig. 4(d) shows that our previous assumption is not without ground. The whole network is a black box whose parameters are updated in training the autoencoder. Nevertheless, we have taken out the intermediate data processed by the two intermediate layers. However, there are no discernible physical meanings in the intermediate representations that can be reasonably interpreted. Therefore, the encoding can be regarded as a pure data compressing operation in this context.

\section{Skeletonized Inversion With 1-D Latent Space}

The misfit functional $J$ for the skeletonized refraction autoencoding inversion is defined as

$$
J=\min _{v(\mathbf{x})} \frac{1}{2} \sum_{s} \sum_{r}\left(\Delta z\left(\mathbf{x}_{r}, \mathbf{x}_{s}\right)\right)^{2}
$$




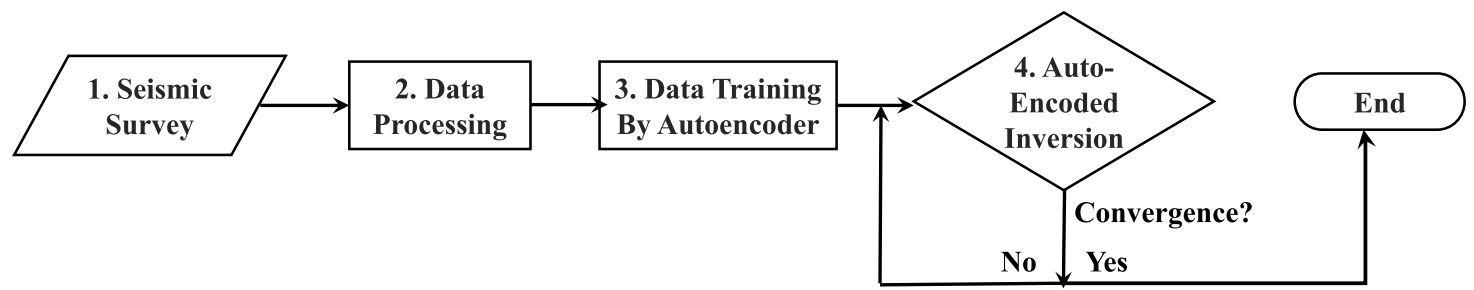

Fig. 5. Workflow of AE inversion.

a)

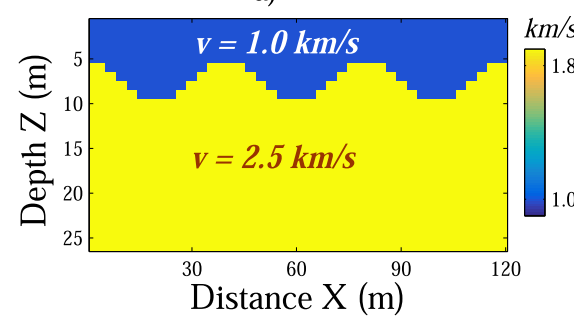

b)

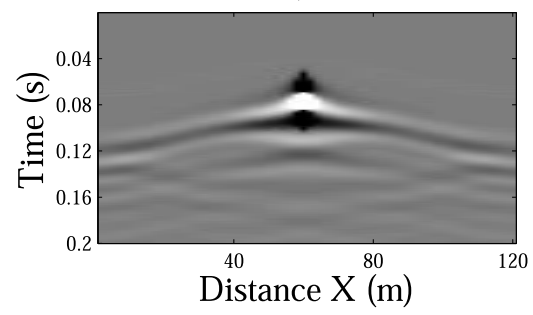

Fig. 6. (a) True velocity model. (b) 30th CSG.

where $\Delta z$ denotes the differences of the encoded values in the 1-D latent space between the observed and the predicted data, namely $\Delta z\left(\mathbf{x}_{r}, \mathbf{x}_{s}\right)=z_{\text {obs }}\left(\mathbf{x}_{r}, \mathbf{x}_{s}\right)-z_{\text {pred }}\left(\mathbf{x}_{r}, \mathbf{x}_{s}\right)$. A source is at location $\mathbf{x}_{s}$, whereas a receiver is at location $\mathbf{x}_{r}$ to record the signals excited from this source. Next, the gradient $\beta$ with respect to the velocity model $v(\mathbf{x})$ for each image point $x$ can, thus, be calculated by

$$
\beta(x)=-\sum_{s} \sum_{r} \frac{\partial \Delta z}{\partial v(x)} \Delta z\left(\mathbf{x}_{r}, \mathbf{x}_{s}\right) .
$$

In a few cases, the skeletonized functional $J$ may converge well, even to the global minimum with the gradient $\beta(\mathbf{x})$, if the true model/structure is not too complex. In principle, if the dimension of the latent space is reduced to one, then there should be no more cycle-skipping phenomenon because all the waveform signals disappear in the misfit functional. However, the annoying local minima problem can still occur during the inversion due to possible reasons, such as highly nonlinear relationship between the compressed data and the true model, unexpected noise in the data, and imperfect inversion methods.

In general, the AE inversion can be guided by four major steps, as shown in Fig. 5.

Step 1 (Seismic Survey): Either forward modeling or field work is used to generate raw data.

Step 2 (Data Processing): First arrivals are roughly windowed and extracted for computing their envelopes.

Step 3 (Data Training): An autoencoder is a setup with a pair of encoder and decoder networks, where each of them contains an equal number of layers with weighting matrices and bias vectors. Guided by (1), the computed envelopes are fed into the autoencoder for training. These trained parameters as weighting matrices and bias vectors are saved for the inversion.

Step 4 (Inversion): Equations (3) and (4) are used to iteratively invert the latent-space misfit function for the velocity model until convergence.
In detail, the Fréchet derivative of $\Delta z$ with respect to $v(x)$ can be calculated using the connective function theory [17]. Here, we tend to use the first arrival envelope instead of the first break in WT as the independent variable since the amplitude together with the phase information, represented by this single encoded scalar, can be more informative about the subsurface structures. From another perspective, the trained autoencoding network simulates a series of linearly independent vectors as a basis, whereas the latent space scalars are like coordinates under this basis. The connective function should quantify a relationship between the velocity field perturbation and the perturbation of the encoded latent variables.

\section{Connective Function}

To invert the first arriving waveforms, we assume the constant-density acoustic wave equation

$$
\frac{1}{v^{2}(\mathbf{x})} \frac{\partial^{2} p\left(\mathbf{x}, t \mid \mathbf{x}_{s}\right)}{\partial t^{2}}-\nabla^{2} p\left(\mathbf{x}, t \mid \mathbf{x}_{s}\right)=\operatorname{src}\left(\mathbf{x}_{s}, t\right)
$$

where $p\left(\mathbf{x}, t \mid \mathbf{x}_{s}\right)$ represents the first arriving pressure field at position $\mathbf{x}$ and time $t$ with a point source excited at $\mathbf{x}_{s}$, and $\operatorname{src}\left(\mathbf{x}_{s}, t\right)$ is the source function. In a 2-D space, the vector $\mathbf{x}$ consists of two components for the horizontal and the vertical coordinates. The solution to (5) is computed by following a finite-difference method [29] and can be written in terms of its Green's function $g\left(\mathbf{x}, t \mid \mathbf{x}^{\prime}, 0\right)$ as:

$$
p\left(\mathbf{x}, t \mid \mathbf{x}_{s}\right)=\int g\left(\mathbf{x}, t \mid \mathbf{x}^{\prime}, 0\right) \star \operatorname{src}\left(\mathbf{x}^{\prime}, t \mid \mathbf{x}_{s}\right) d \mathbf{x}^{\prime}
$$

where the symbol $\star$ denotes convolution in time.

Let $e_{z}\left(\mathbf{x}_{r}, t ; \mathbf{x}_{s}\right)$ denote the envelope of a trace $p\left(\mathbf{x}_{r}, t \mid \mathbf{x}_{s}\right)$ recorded at $\mathbf{x}_{r}$ associated with the source at $\mathbf{x}_{s}$. A correlation function $f$ is defined as the function that connects the skeletonized data with the recorded pressure field. Its form is

$$
f\left(\mathbf{x}_{r}, z ; \mathbf{x}_{s}\right)=\int \frac{e_{\mathrm{obs}, z^{*}+z}\left(\mathbf{x}_{r}, t ; \mathbf{x}_{s}\right)}{A_{\mathrm{obs}}\left(\mathbf{x}_{r}, t ; \mathbf{x}_{s}\right)} e_{\mathrm{pred}, z^{*}}\left(\mathbf{x}_{r}, t ; \mathbf{x}_{s}\right) d t
$$


a)

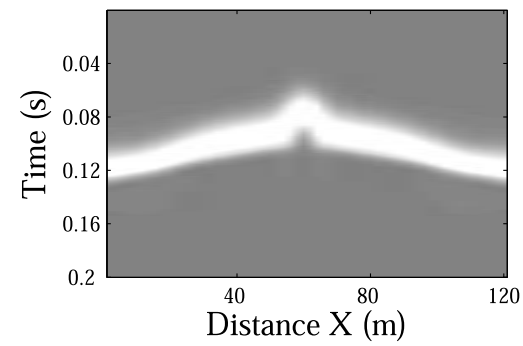

b)

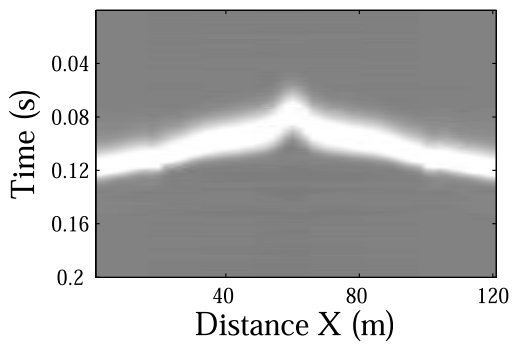

c)

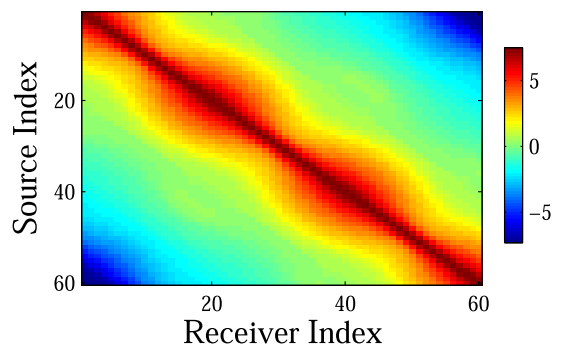

Fig. 7. (a) Envelope of first arrivals in the 30th CSG (b) its decoded envelope. (c) Matrix latent-space values for all the traces (encoding results).

a)

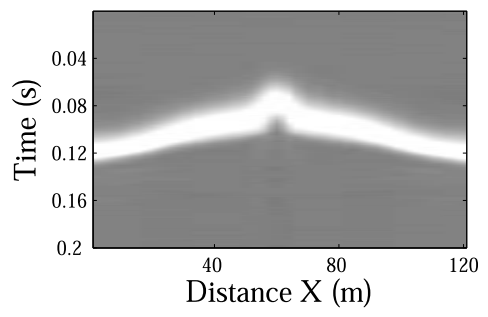

b)

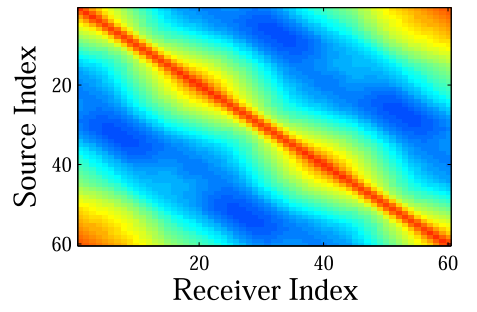

c)

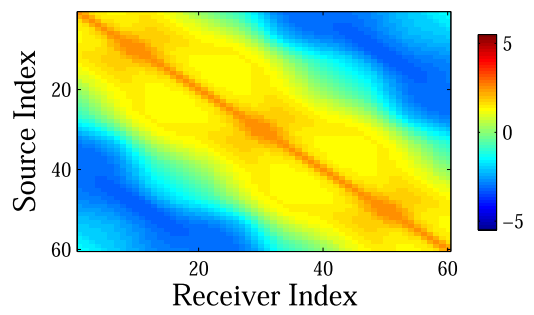

Fig. 8. (a) Decoded envelope of CSG \#30 with 2-D latent space. (b) First- and (c) second-dimensional latent-space values for all the traces (encoding results).

where $z^{*}$ denotes the encoded value of the calculated envelope $e_{\text {pred }, z^{*}}\left(\mathbf{x}_{r}, t ; \mathbf{x}_{s}\right)$ with its later arrivals muted. This subscript $z^{*}$ is the scalar representing its skeletonized feature encoded by a well-trained autoencoder neural network. The denominator $A_{\mathrm{obs}}\left(\mathbf{x}_{r}, t ; \mathbf{x}_{s}\right)$ represents the maximum amplitude of $e_{\mathrm{obs}, z^{*}+z}\left(\mathbf{x}_{r}, t ; \mathbf{x}_{s}\right)$. In the same way, $e_{\mathrm{obs}, z^{*}+z}\left(\mathbf{x}_{r}, t ; \mathbf{x}_{s}\right)$ represents the envelope of an observed trace merely with its first arrivals encoded as $z^{*}+z$. The observed and predicted envelopes share the same source and receiver locations. Here, the two subscripts $z^{*}+z$ and $z^{*}$ represent two encoded envelopes of an observed trace and a calculated trace, respectively. These two expressions are used simply for indicting that $z^{*}$ is relatively fixed, and $z$ denotes the real difference between the two signals in the latent space. In other words, to make the two envelopes match or equal in the new domain, the operation of changing $z$ can be seen as shifting the observed envelope in WT in the time domain to make it match the predicted data. Also, similar to building the correlation function in WT, if the function of the two envelopes in (7) reaches its maximum, then there must exist a $\Delta z$ with $z=$ $\Delta z$ depicting their difference in the latent space. Therefore, by taking the derivative of $f$ with respect to $z$, we have

$$
\begin{aligned}
\dot{f}_{\Delta z} & =\left.\frac{\partial f\left(\mathbf{x}_{r}, z ; \mathbf{x}_{s}\right)}{\partial z}\right|_{z=\Delta z} \\
& =\int \frac{\dot{e}_{\text {obs }, z^{*}+\Delta z}\left(\mathbf{x}_{r}, t ; \mathbf{x}_{s}\right)}{A_{\text {obs }}\left(\mathbf{x}_{r}, t ; \mathbf{x}_{s}\right)} e_{\text {pred }, z^{*}}\left(\mathbf{x}_{r}, t ; \mathbf{x}_{s}\right) d t=0 .
\end{aligned}
$$

Here, the derivative, as the dot operator above $f$, taken on the first term of the right-hand side of (8) is

$$
\dot{e}_{\mathrm{obs}, z^{*}+\Delta z}\left(\mathbf{x}_{r}, t ; \mathbf{x}_{s}\right)=\left.\frac{\partial e_{\mathrm{obs}, z^{*}+\Delta z}\left(\mathbf{x}_{r}, t ; \mathbf{x}_{s}\right)}{\partial z}\right|_{z=\Delta z} .
$$

In terms of computation, (9) presents the reason why a decoder is required because the derivative of an envelope with respect to $z$ can be numerically approximated using the decoder,

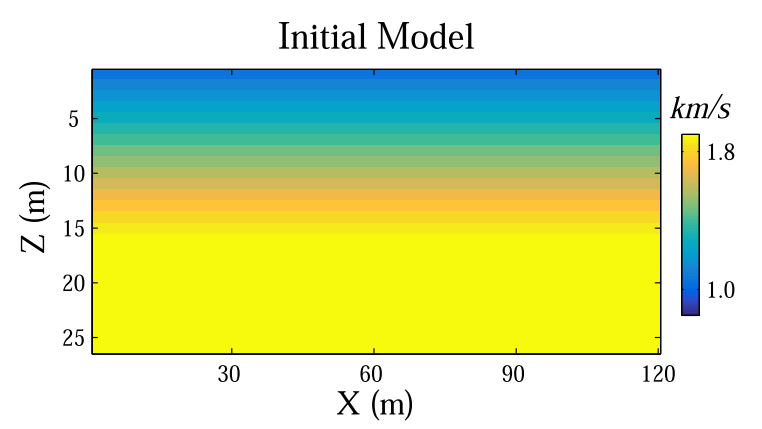

Fig. 9. Starting gradient model.

which is a very important procedure in this method. Using the implicit function theorem, the Fréchet derivative in (4) can be expanded as

$$
\begin{aligned}
\frac{\partial \Delta z}{\partial v(x)} & =-\left(\frac{\partial \dot{f}_{\Delta z}}{\partial v(x)}\right)\left(\frac{\partial \dot{f}_{\Delta z}}{\partial \Delta z}\right)^{-1} \\
& =-\frac{1}{E} \int \dot{e}_{\mathrm{obs}, z^{*}+\Delta z}\left(\mathbf{x}_{r}, t ; \mathbf{x}_{s}\right) \frac{\partial e_{\mathrm{pred}, z^{*}}\left(\mathbf{x}_{r}, t ; \mathbf{x}_{s}\right)}{\partial v(x)} d t
\end{aligned}
$$

where the denominator can be written as

$$
E=\int \ddot{e}_{\mathrm{obs}, z^{*}+\Delta z}\left(\mathbf{x}_{r}, t ; \mathbf{x}_{s}\right) e_{\text {pred }, z^{*}}\left(\mathbf{x}_{r}, t ; \mathbf{x}_{s}\right) d t
$$

In (10), the Fréchet derivative as the gradient image for updating the velocity field is

$$
\begin{aligned}
& \frac{\partial e_{\text {pred }, z^{*}}\left(\mathbf{x}_{r}, t ; \mathbf{x}_{s}\right)}{\partial v(x)} \\
& =\frac{1}{\sqrt{p_{\text {pred } z^{*}}^{2}\left(\mathbf{x}_{r}, t ; \mathbf{x}_{s}\right)+h p_{\text {pred }, z^{*}}^{2}\left(\mathbf{x}_{r}, t ; \mathbf{x}_{s}\right)}} \\
& \quad \times\left[p_{\text {pred }, z^{*}}\left(\mathbf{x}_{r}, t ; \mathbf{x}_{s}\right) \frac{\partial p_{\text {pred } z^{*}}\left(\mathbf{x}_{r}, t ; \mathbf{x}_{s}\right)}{\partial v(x)}\right. \\
& \left.\quad+h p_{\text {pred }, z^{*}}\left(\mathbf{x}_{r}, t ; \mathbf{x}_{s}\right) \frac{\partial h p_{\text {pred }, z^{*}}\left(\mathbf{x}_{r}, t ; \mathbf{x}_{s}\right)}{\partial v(x)}\right]
\end{aligned}
$$


a)

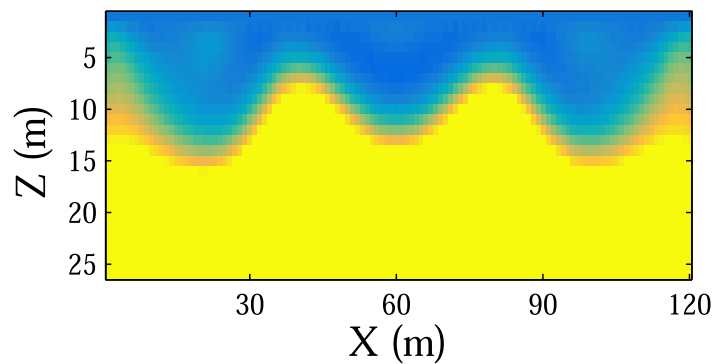

c)

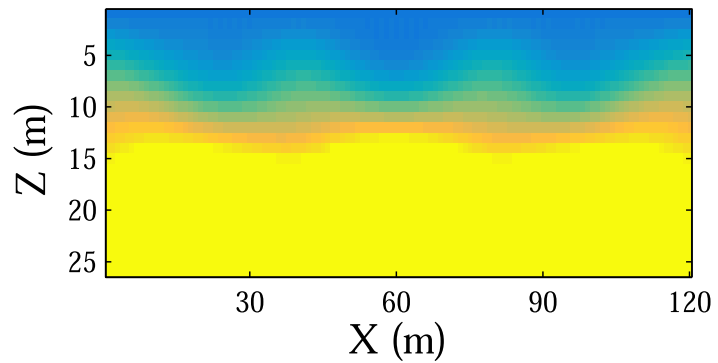

e)

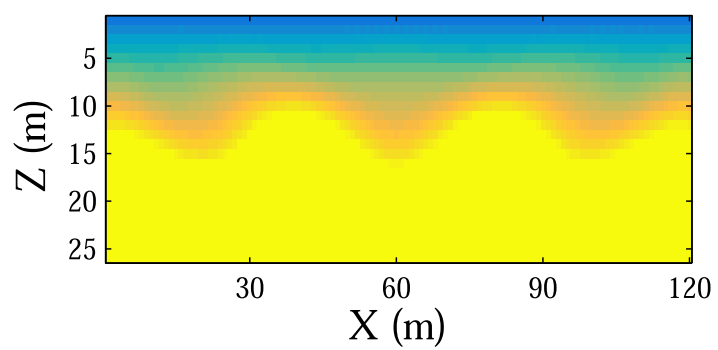

b)

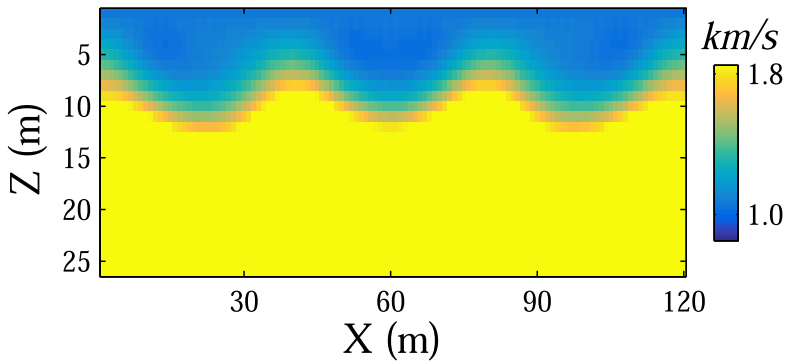

d)

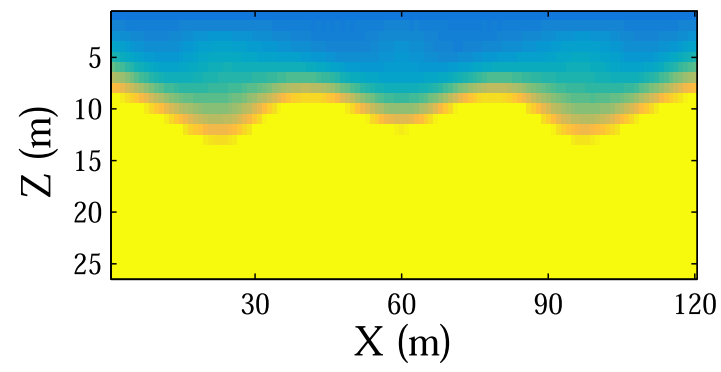

Fig. 10. Tomograms inverted for 50 iterations by $30 \mathrm{~Hz}$. (a) WT inverted model. (b) Skeletonized AE inversion (AE inverted model with 1-D latent space). (c) Envelope inversion model. (d) AE inverted model with 2-D latent space. (e) FWI inverted model.

where $h p$ means the pure Hilbert transform of the raw predicted pressure field profile $p$ without taking the envelope. Moreover, in detail, we have

$$
\begin{gathered}
\frac{\partial p_{\text {pred }, z^{*}}\left(\mathbf{x}_{r}, t ; \mathbf{x}_{s}\right)}{\partial v(x)}=\frac{2}{v^{3}(x)} \dot{g}\left(x, t ; \mathbf{x}_{\mathbf{r}}, 0\right) \star \dot{p}_{\text {pred }, z^{*}}\left(x, t ; \mathbf{x}_{s}\right), \\
\frac{\partial h p_{\text {pred }, z^{*}}\left(\mathbf{x}_{r}, t ; \mathbf{x}_{s}\right)}{\partial v(x)}=\frac{2}{v^{3}(x)} \dot{g}\left(x, t ; \mathbf{x}_{\mathbf{r}}, 0\right) \star \dot{h} \dot{p}_{\text {pred }, z^{*}}\left(x, t ; \mathbf{x}_{s}\right)
\end{gathered}
$$

where $g$ denotes the backpropagated Green's function for the acoustic wave-equation, and the dot operator means the derivative with respect to time. Equations (13) and (14) are nothing but to conduct two RTMs. As (12) represents the standard Fréchet derivative of the envelope with respect to the velocity variations, we will also compare the results of the proposed method with the envelope inversion results in the next numerical experiment section. Inserting (10)-(14) to (4), we have the ultimate gradient expression. In this study, the gradient of refraction AE inversion for the latentspace misfit function is derived using our notation similar to that in [23]. Its final form is given as

$$
\begin{aligned}
& \beta(x) \\
& =-\sum_{s} \sum_{r} \frac{\partial \Delta z}{\partial v(x)} \Delta z\left(\mathbf{x}_{r}, \mathbf{x}_{s}\right) \\
& =\frac{1}{v^{3}(x)} \sum_{s} \sum_{r}\left\{\frac { \Delta z ( \mathbf { x } _ { r } , \mathbf { x } _ { s } ) } { E } \int d t \left[\dot{e}_{\mathrm{obs}, z^{*}+\Delta z}\left(\mathbf{x}_{r}, t ; \mathbf{x}_{s}\right)\right.\right. \\
& \quad \times \frac{1}{\sqrt{p_{\text {pred } z^{*}}^{2}\left(\mathbf{x}_{r}, t ; \mathbf{x}_{s}\right)+h p_{\text {pred }, z^{*}}^{2}\left(\mathbf{x}_{r}, t ; \mathbf{x}_{s}\right)}} \\
& \quad \times\left(p_{\text {pred } z^{*}}\left(\mathbf{x}_{r}, t ; \mathbf{x}_{s}\right) \dot{g}\left(x, t ; \mathbf{x}_{\mathbf{r}}, 0\right) \star \dot{p}_{\text {pred }, z^{*}}\left(x, t ; \mathbf{x}_{s}\right)\right. \\
& \left.\left.\left.\quad+h p_{\text {pred }, z^{*}}\left(\mathbf{x}_{r}, t ; \mathbf{x}_{s}\right) \dot{g}\left(x, t ; \mathbf{x}_{\mathbf{r}}, 0\right) \star \dot{h p_{\text {pred }, z^{*}}}\left(x, t ; \mathbf{x}_{s}\right)\right)\right]\right\} .
\end{aligned}
$$

Now, the velocity tomogram $v(x)$ can be iteratively updated by a preconditioned conjugated gradient method [30] as

$$
v_{k+1}(x)=v_{k}(x)+\alpha_{k} \beta_{k}(x)
$$

where $k$ indexes the iteration number, and the step-length $\alpha_{k}$ is computed using a quadratic approximation. 
a)

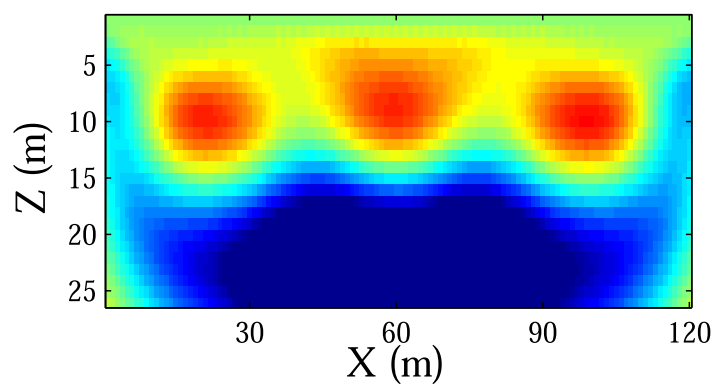

c)

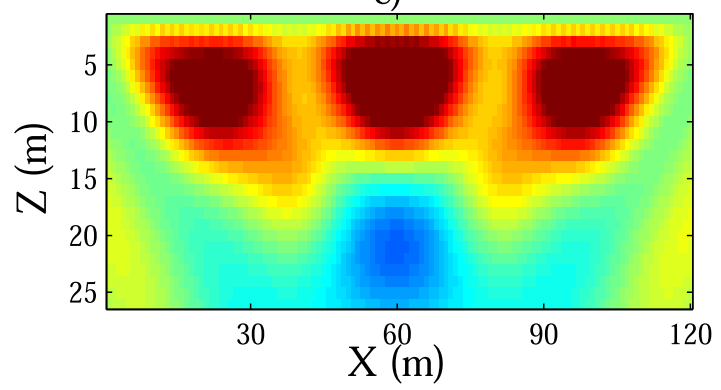

e)

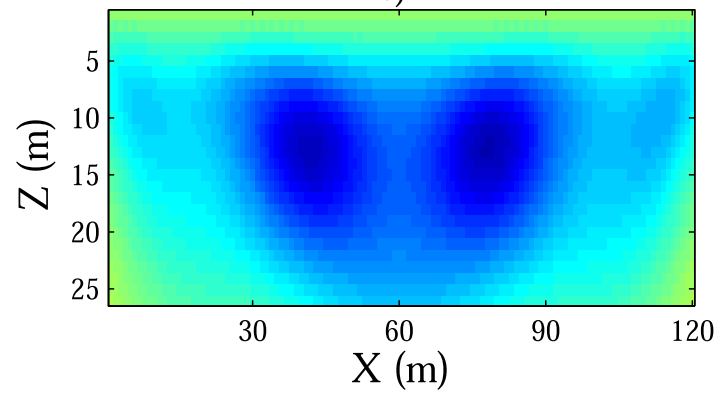

b)

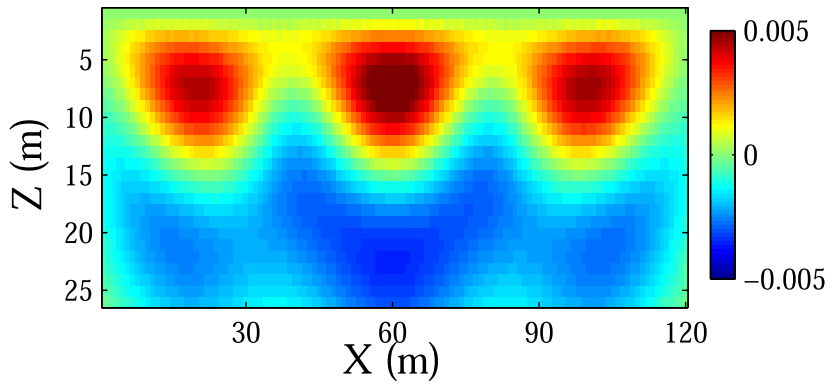

d)

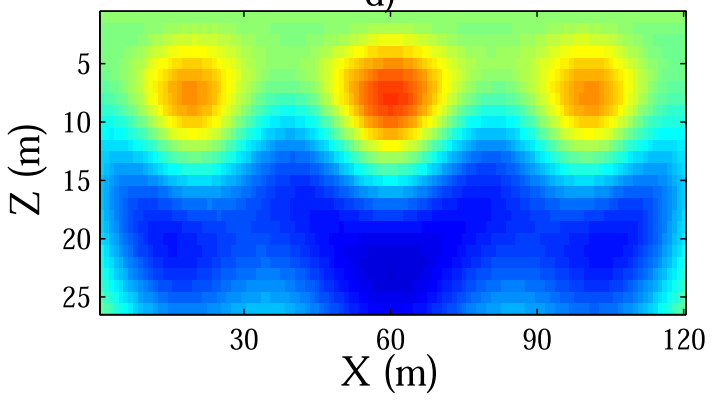

Fig. 11. Gradients computed by (a) WT, (b) skeletonized AE inversion [AE inversion gradient (1-D)], (c) envelope inversion, (d) AE inversion with 2-D latent space, and (e) FWI in the first iteration.

\section{E. Inversion With 2-D Latent Space}

If the dimension of the latent space is set to two as $\mathbf{z}=\left(z_{1}, z_{2}\right)$, then the degree of freedom to express the envelopes is increasing. Therefore, the corresponding inversion theory in [23] needs to be modified with a few changes to the misfit function. The misfit function associated with the 2-D latent space autoencoder can be defined as

$$
L=\min _{v(\mathbf{x})} \frac{1}{2} \sum_{s} \sum_{r}\left(\Delta z_{1}^{2}\left(\mathbf{x}_{r}, \mathbf{x}_{s}\right)+\Delta z_{2}^{2}\left(\mathbf{x}_{r}, \mathbf{x}_{s}\right)\right)
$$

and the gradient image $\gamma(x)$ becomes

$$
\begin{aligned}
\gamma(x) & =-\frac{\partial L}{\partial v(x)} \\
& =-\sum_{s} \sum_{r}\left(\frac{\partial \Delta z_{1}}{\partial v(x)} \Delta z_{1}\left(\mathbf{x}_{r}, \mathbf{x}_{s}\right)+\frac{\partial \Delta z_{2}}{\partial v(x)} \Delta z_{2}\left(\mathbf{x}_{r}, \mathbf{x}_{s}\right)\right)
\end{aligned}
$$

where the velocity distribution $v(x)$ is also updated by

$$
v_{k+1}(x)=v_{k}(x)+\alpha_{k} \gamma_{k}(x) .
$$

Here, we redefine the correlation function $q\left(\mathbf{x}_{r}, z_{1}, z_{2} ; \mathbf{x}_{s}\right)$ by $q\left(\mathbf{x}_{r}, z_{1}, z_{2} ; \mathbf{x}_{s}\right)=\int \frac{e_{\mathrm{obs}, z_{1}^{*}+z_{1}, z_{2}^{*}+z_{2}}\left(\mathbf{x}_{r}, t ; \mathbf{x}_{s}\right)}{A_{\mathrm{obs}}\left(\mathbf{x}_{r}, t ; \mathbf{x}_{s}\right)} e_{\mathrm{pred}, z_{1}^{*}, z_{2}^{*}}\left(\mathbf{x}_{r}, t ; \mathbf{x}_{s}\right) d t$

where the encoded latent-space vector $\left(z_{1}, z_{2}\right)$ denotes the envelope features. When the correlation function $q$ reaches its maximum, taking the gradient of $q$ with respect to $\mathbf{z}=$ $\left(z_{1}, z_{2}\right)$ should be equal to zero as a usual necessary condition. Therefore, we have

$$
\nabla q_{\left(\Delta z_{1}, \Delta z_{2}\right)}=\left(q_{1}, q_{2}\right)=\left.\left(\frac{\partial q}{\partial z_{1}}, \frac{\partial q}{\partial z_{2}}\right)\right|_{\left(z_{1}, z_{2}\right)=\left(\Delta z_{1}, \Delta z_{2}\right)}=0
$$

and, in detail, the two elements can be, respectively, expanded as

$$
\begin{aligned}
q_{1}= & \left.\frac{\partial q}{\partial z_{1}}\right|_{\left(z_{1}, z_{2}\right)=\left(\Delta z_{1}, \Delta z_{2}\right)}=0 \\
= & \int \frac{e_{\mathrm{pred}, z_{1}^{*}, z_{2}^{*}\left(\mathbf{x}_{r}, t ; \mathbf{x}_{s}\right)}}{A_{\mathrm{obs}}\left(\mathbf{x}_{r}, t ; \mathbf{x}_{s}\right)} \\
& \times\left.\frac{\partial e_{\mathrm{obs}, z_{1}^{*}+z_{1}, z_{2}^{*}+z_{2}}\left(\mathbf{x}_{r}, t ; \mathbf{x}_{s}\right)}{\partial z_{1}}\right|_{\left(z_{1}, z_{2}\right)=\left(\Delta z_{1}, \Delta z_{2}\right)} d t
\end{aligned}
$$


a)

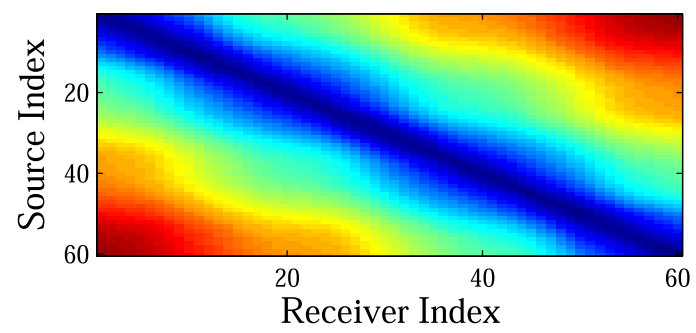

c)

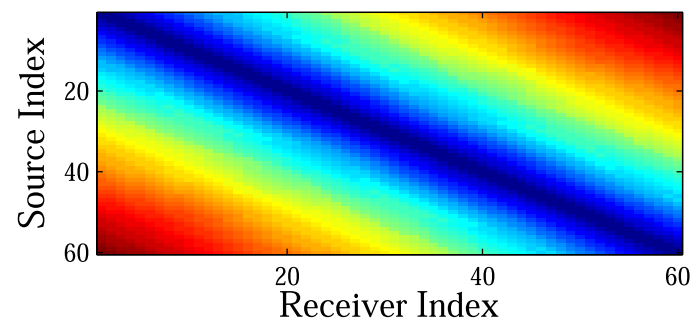

e)

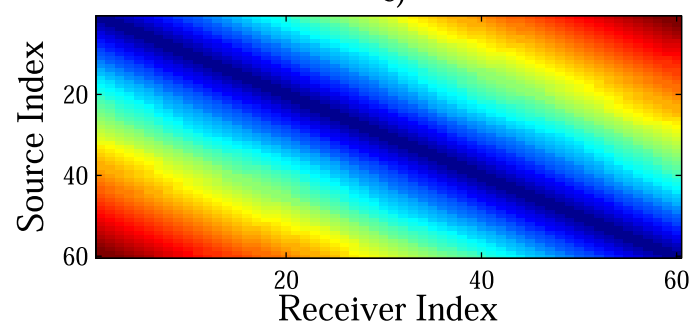

b)

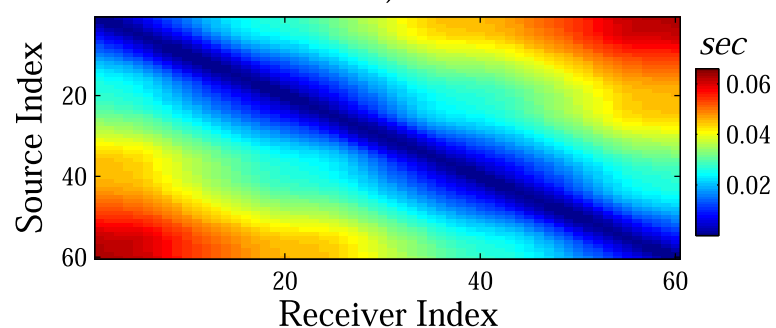

d)

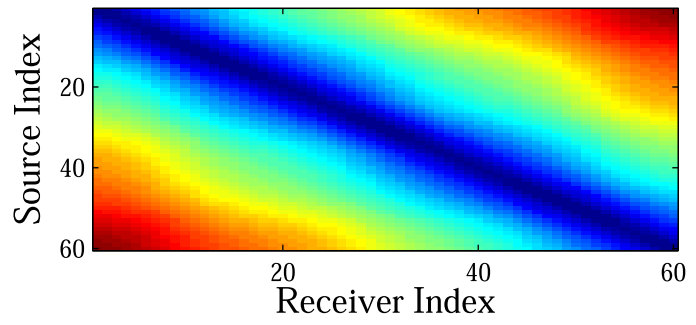

f)

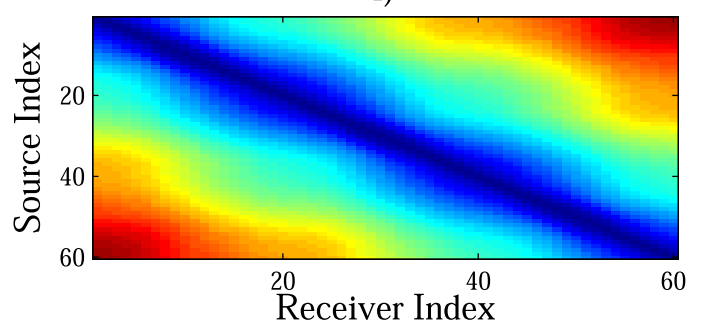

Fig. 12. First-arrival travel-time matrices of tomograms inverted by (a) WT, (b) AE inversion (1-D), (c) envelope inversion, (d) AE inversion (2-D), and (e) FWI. (f) True first arrival matrix.

a)

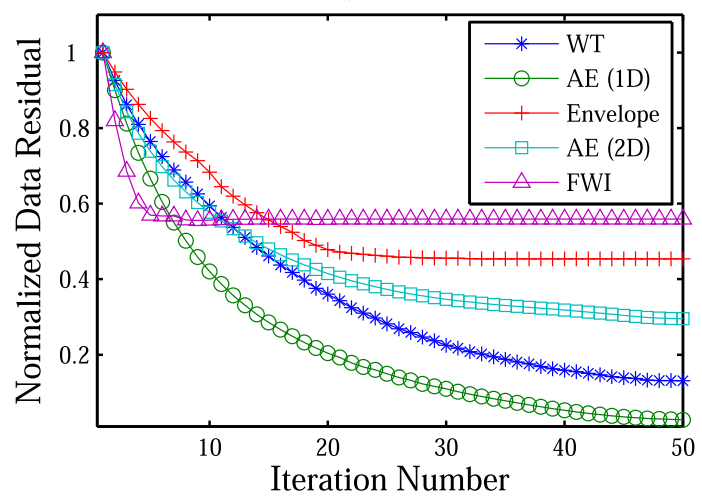

b)

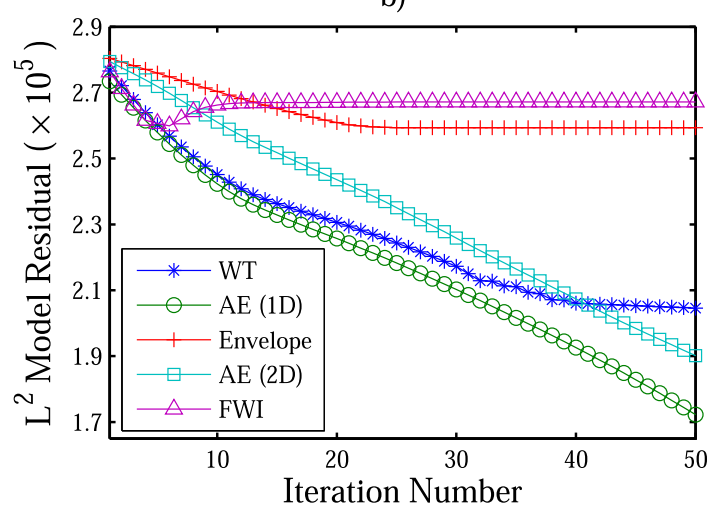

Fig. 13. Comparisons of (a) data and (b) model residuals above the depth $Z=17 \mathrm{~m}$ with five different methods. (a) Variations of data residuals. (b) Variations of inverted model residuals.

and

$$
\begin{aligned}
q_{2}= & \left.\frac{\partial q}{\partial z_{2}}\right|_{\left(z_{1}, z_{2}\right)=\left(\Delta z_{1}, \Delta z_{2}\right)}=0 \\
= & \int \frac{e_{\mathrm{pred}, z_{1}^{*}, z_{2}^{*}\left(\mathbf{x}_{r}, t ; \mathbf{x}_{s}\right)}}{A_{\mathrm{obs}}\left(\mathbf{x}_{r}, t ; \mathbf{x}_{s}\right)} \\
& \times\left.\frac{\partial e_{\mathrm{obs}, z_{1}^{*}+z_{1}, z_{2}^{*}+z_{2}\left(\mathbf{x}_{r}, t ; \mathbf{x}_{s}\right)}}{\partial z_{2}}\right|_{\left(z_{1}, z_{2}\right)=\left(\Delta z_{1}, \Delta z_{2}\right)} d t .
\end{aligned}
$$

If we differentiate (22) and (23) once more with respect to $\Delta z_{1}, \Delta z_{2}$, and $v(x)$, then they are

$$
\frac{\partial^{2} q}{\partial \Delta z_{1}^{2}} d \Delta z_{1}+\frac{\partial^{2} q}{\partial \Delta z_{1} \partial \Delta z_{2}} d \Delta z_{2}+\frac{\partial^{2} q}{\partial \Delta z_{1} \partial v(x)} d v=0
$$

and

$\frac{\partial^{2} q}{\partial \Delta z_{2} \partial \Delta z_{1}} d \Delta z_{1}+\frac{\partial^{2} q}{\partial \Delta z_{2}^{2}} d \Delta z_{2}+\frac{\partial^{2} q}{\partial \Delta z_{2} \partial v(x)} d v=0$. 
a)

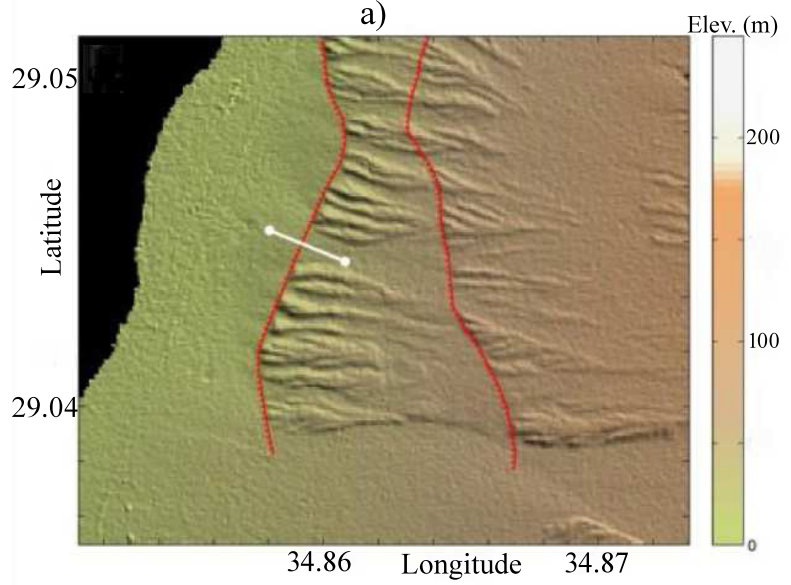

b)

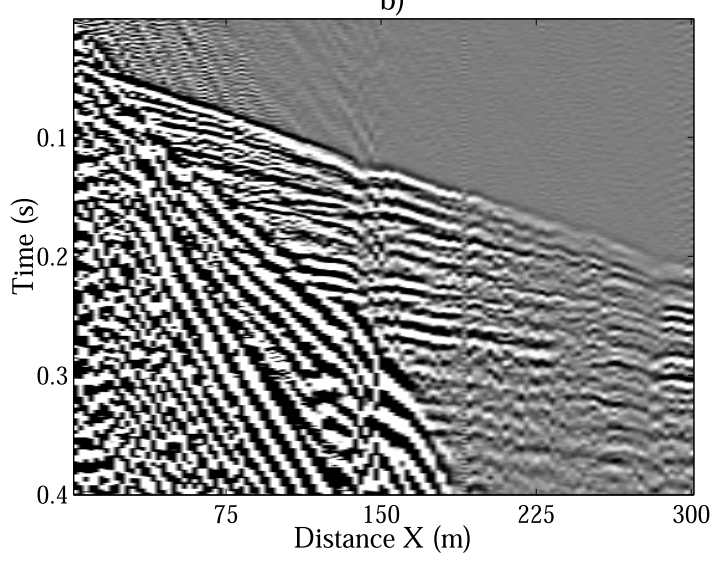

Fig. 14. (a) Aqaba data acquisition map with the white seismic line (topographic map of Aqaba gulf). (b) Raw CSG \#1 (Aqaba data CSG \#1).

a)

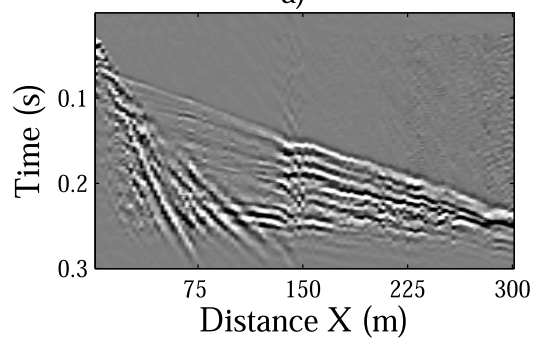

b)

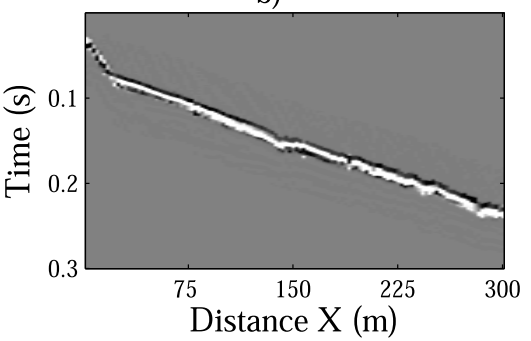

Fig. 15. (a) Processed CSG \#1 and (b) its first arrivals.

a)

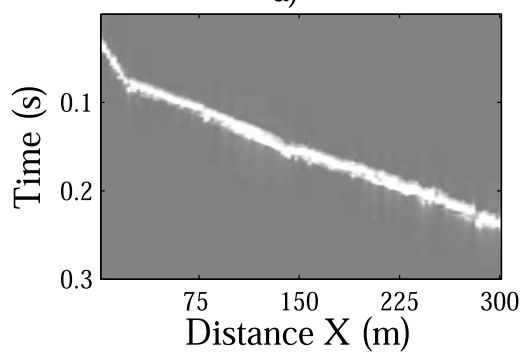

b)

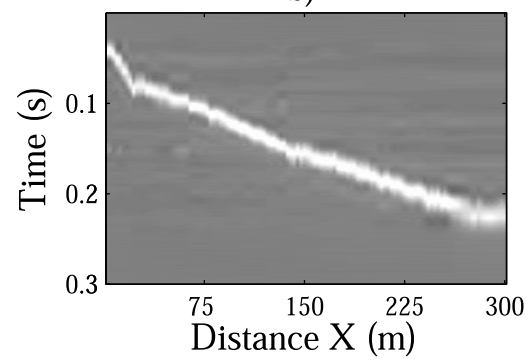

c)

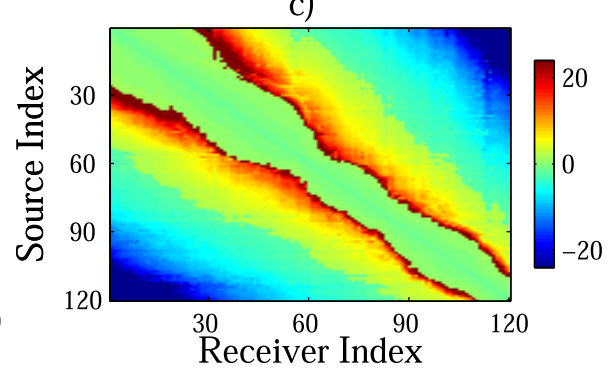

Fig. 16. (a) Envelope of first arrival CSG \#1 (b) its decoded envelope (1-D) and (c) encoded latent space of all the traces (Aqaba data).

To obtain the two gradient terms in (18), we solve (24) and (25) by

$$
\left[\begin{array}{l}
\frac{\partial \Delta z_{1}}{\partial v(x)} \\
\frac{\partial \Delta z_{2}}{\partial v(x)}
\end{array}\right]=-\left[\begin{array}{ll}
q_{11} & q_{12} \\
q_{21} & q_{22}
\end{array}\right]^{-1}\left[\begin{array}{c}
\frac{\partial^{2} q}{\partial \Delta z_{1} \partial v(x)} \\
\frac{\partial^{2} q}{\partial \Delta z_{2} \partial v(x)}
\end{array}\right] .
$$

Here, the partial derivative of $q$ about $v(x)$ is taken on the predicted envelope term as the regular Fréchet derivative in (13) and (14). Therefore, the two imaging elements are computed by

$$
\begin{aligned}
\frac{\partial^{2} q}{\partial \Delta z_{j} \partial v}=\int \frac{1}{A_{\mathrm{obs}}\left(\mathbf{x}_{r}, t ; \mathbf{x}_{s}\right)} \frac{\partial e_{\mathrm{pred}, z_{1}^{*}, z_{2}^{*}\left(\mathbf{x}_{r}, t ; \mathbf{x}_{s}\right)}^{\partial v(x)}}{} & \frac{\partial e_{\mathrm{obs}, z_{1}^{*}+\Delta z_{1}, z_{2}^{*}+\Delta z_{2}}\left(\mathbf{x}_{r}, t ; \mathbf{x}_{s}\right)}{\partial \Delta z_{j}} d t
\end{aligned}
$$

where $j=1,2$. The terms $q_{11}$ and $q_{22}$ are the secondorder derivatives on $\Delta z_{1}$ and $\Delta z_{2}$, respectively. Similarly, $q_{12}$ and $q_{21}$ denote two second-order mixed derivatives on $\Delta z_{1}$ and $\Delta z_{2}$, and we assume that $q_{12}=q_{21}$ in this study without loss of generality.

\section{NumericAl TESTS}

The AE (or NML) method is tested for a land survey using both synthetic data and field data. The field data are from a seismic survey near the Gulf of Aqaba in a desert environment.

\section{A. Synthetic Test on Sinusoid Model}

The model shown in Fig. 6(a) is used to generate synthetic data to test the effectiveness of the proposed method. The model size is $26 \mathrm{~m} \times 120 \mathrm{~m}$ in the vertical and horizontal 
a)

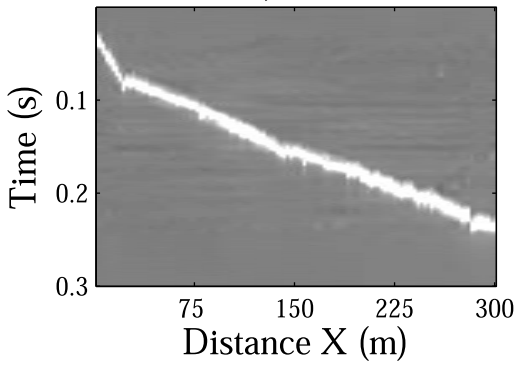

b)

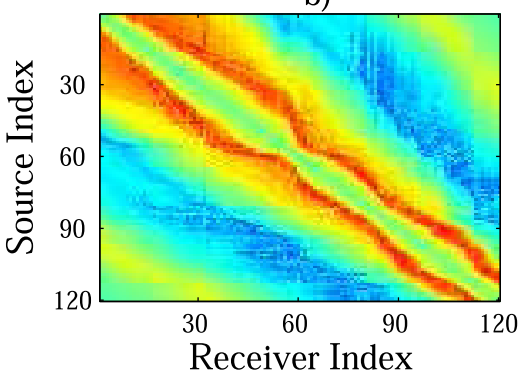

c)

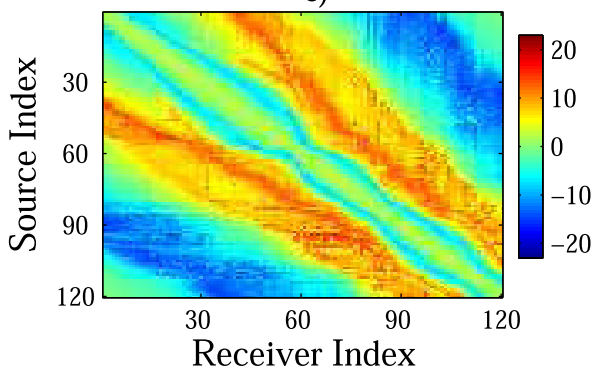

Fig. 17. (a) Decoded envelope of first arrival CSG \#1 with 2-D latent space. (b) First- and (c) second-dimensional latent-space values for all the traces (encoding results).

directions, respectively. The data are recorded by 60 receivers spaced at an interval of $2 \mathrm{~m}$, and they are triggered by 60 sources at every receiver's location. A grid point of spacing of $1 \mathrm{~m}$ is set for both the horizontal and the vertical directions. Based on the acoustic wave equation (5), a Ricker wavelet with a frequency band peaked at $30 \mathrm{~Hz}$ is excited as the source to conduct the forward modeling for all the CSGs, and a typical CSG \#30 is shown in Fig. 6(b). The recording time for each CSG is $0.2 \mathrm{~s}$ with a sampling interval of $0.1 \mathrm{~ms}$, so the number of temporal samples is set as $M=2000$ for each trace. Four types of methods are, respectively, applied to reconstruct the subsurface velocity models for comparisons. The first is WT inversion; the second is the proposed skeletonized AE inversion method in this study; the third one is the envelope inversion method introduced by $\mathrm{Wu}$ and Chen [11]; and the fourth one is the conventional FWI for a comparison. Moreover, the skeletonized AE inversion with 2-D latent space is also applied for comparisons. The detailed steps to carry out our method with the previous theoretical section are given in the following.

Step 1: The first breaks are automatically windowed out with a rough estimate.

Step 2: The first arrivals in all the traces are extracted with a temporal window size of 120 samples starting from the first breaks; the later arrivals outside the time window are gradually muted by a decaying exponential function for avoiding the frequency leakage problem.

Step 3: Envelopes of all the first arrivals are computed and then fed into the autoencoding neural networks for training. Here, the hyperparameters are Dim1 = 2000, $\operatorname{Dim} 2=500$, and Dim $3=90$.

Step 4: Skeletonized AE inversions are carried out based on (3), (4), and (16)-(19) for the subsurface velocity distributions.

The envelopes calculated in Step 3 can efficiently be used for the envelope inversion. Here, sizes of the three encoding weighting matrices $W_{1}, W_{2}$, and $W_{3}$ are $2000 \times 500,500 \times$ 90 , and $90 \times 1$ (or 2), respectively. Accordingly, sizes of the three decoding matrices $W_{1}^{\prime}, W_{2}^{\prime}$, and $W_{3}^{\prime}$ are $500 \times 2000$, $90 \times 500$, and 1 (or 2) $\times 90$ in a reverse order. As an example, the envelopes of first arrivals in CSG \#30 are presented in Fig. 7(a), which are encoded and decoded in turn with the output shown in Fig. 7(b). The learning rate is set up as 0.001 , and the iteration number is set as 50 . These empirical

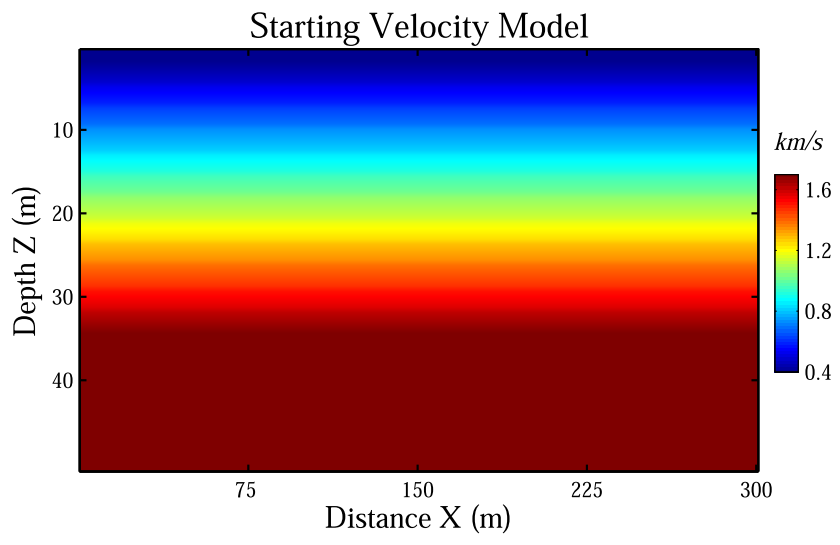

Fig. 18. Starting model.

parameters, for this simple example, are obtained by trial and error to ensure the recovery of all the envelope signals. The entire latent space values are finally saved in a matrix, as illustrated in Fig. 7(c). In the case of mapping the envelopes into 2-D latent space, only the lowest dimension is set to two, and other preset parameters are identical to the 1-D case. Fig. 8(a) presents the decoded envelope of CSG \#30, which is superior to the 1-D case shown in Fig. 7(b) by referring to the standard envelope in Fig. 7(a). Fig. 8(b) and (c) presents the two encoded latent-space matrices for all the traces in this data set.

With the initial gradient model presented in Fig. 9, the abovementioned four methods and FWI are used to invert the velocity model. For fairness in the following comparisons, all these inversions are applied to the same first arriving CSGs. After 50 iterations when all the data misfit functionals stop decreasing, the inverted models are shown in Fig. 10(a)-(e), implying the advantages of the proposed method because Fig. 10(b) and 10(d) is closer to the true model in Fig. 6(a) than other three tomograms. Five gradient images of the first iteration are also presented in Fig. 11(a)-(e), which reveals that the skeletonized autoencoding inversion (both 1-D and 2-D) can reconstruct the essential features of the first arriving signals. For a sanity test, all the first breaks picked from the CSGs associated with the five inverted models and the true model are, respectively, presented in Fig. 12(a)-(f). Fig. 12(b) and (d) again illustrates the advantages of this method over the other three methods in this case. Note that both the WT tomogram 
a)

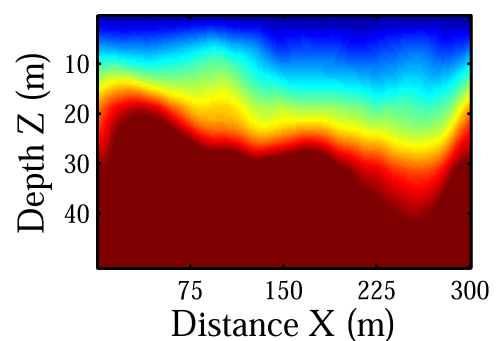

d)

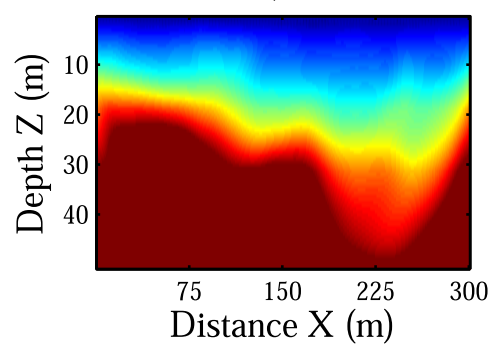

b)

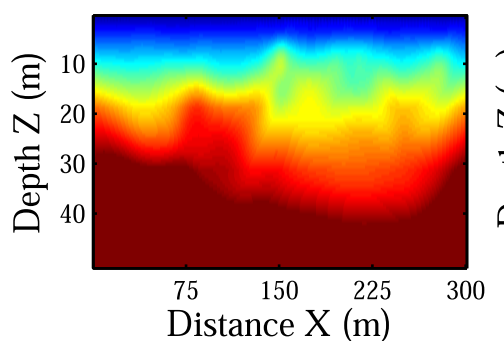

e)

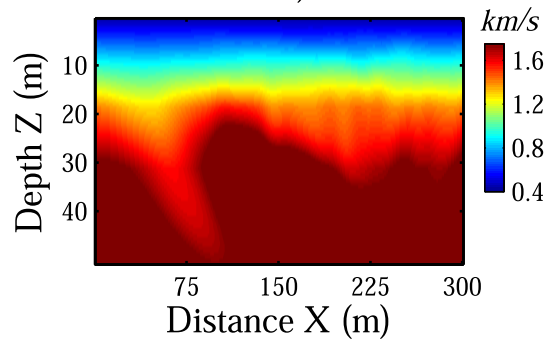

c)

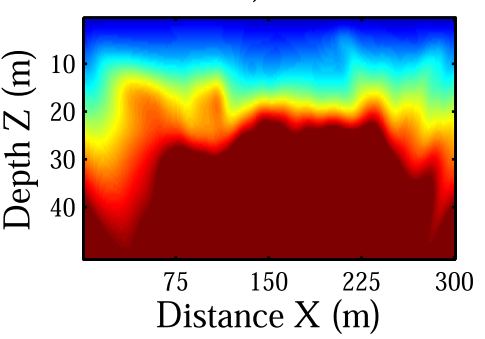

Distance X (m)

Fig. 19. Tomograms inverted by (a) 50-Hz WT, (b) 50-Hz skeletonized AE inversion, (c) envelope inversion, (d) AE inversion with 2-D latent space, and (e) FWI.

a)

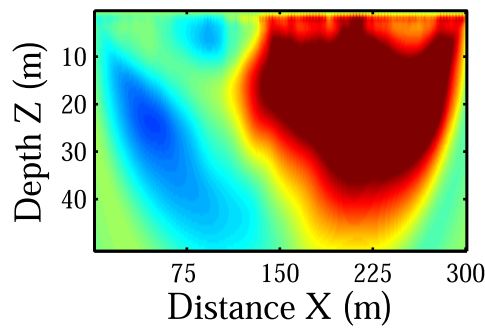

d)

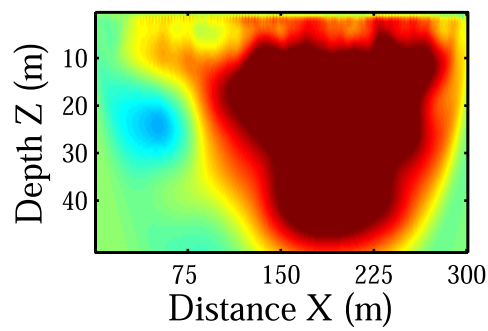

b)

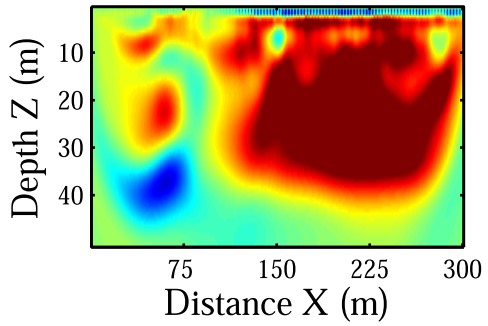

e)

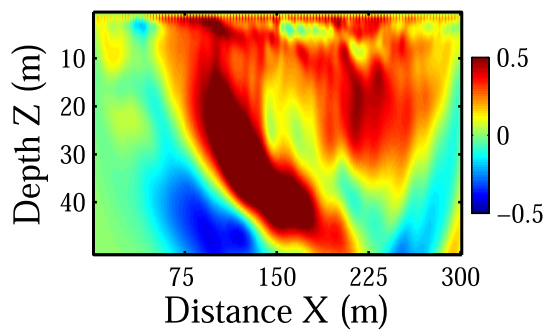

Fig. 20. Gradients inverted by (a) WT, (b) skeletonized AE inversion, (c) envelope inversion, (d) AE inversion with 2-D latent space, and (e) FWI in the first iteration.

[see Fig. 10(a)] and its corresponding first arrival matrix [see Fig. 12(a)] show that this reconstructed interface oscillates stronger than the true interface in Fig. 6(a) although the general basic structure can be rebuilt.

For quantitative comparisons, Fig. 13(a) and (b) presents the data misfit and the model misfit curves with all the five algorithms for the first 50 iterations. As expected, if the number of local minima decreases with less amount of the input data, inversions can achieve better convergence rates because the observed data can be more easily approximated. To avoid the overfitting problems, all the iterations are terminated if their objective functions cease to decrease because they are all defined in the data domain. In Fig. 13(a), both WT and the $\mathrm{AE}$ inversions (1-D and 2-D) converge faster than the envelope inversion and FWI, and the latter two inversions cease to update the models after several iterations possibly due to the local minima problems. The AE inversions perform better than the envelope inversion or FWI in which the Fréchet derivatives in (15) and (18) are weighted by the terms $\Delta z$ and $\left(\Delta z_{1}, \Delta z_{2}\right)$, measuring the distance between traces in the latent space, but not in the raw data space.

Another criterion is the model misfit function that can directly measure the quality of inverted tomograms although manual visual recognitions are often indispensable as an auxiliary technique for quality control. In Fig. 13(b), the tomogram computed by the $\mathrm{AE}$ inversion (1-D) is the closest to the 
a)

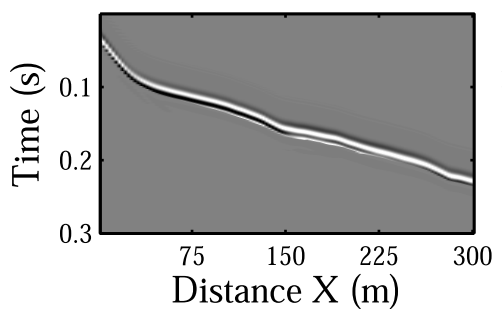

d)

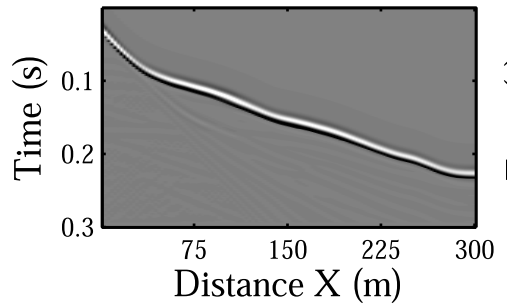

b)

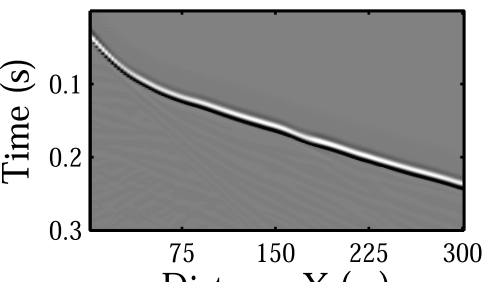

Distance X (m)

e)

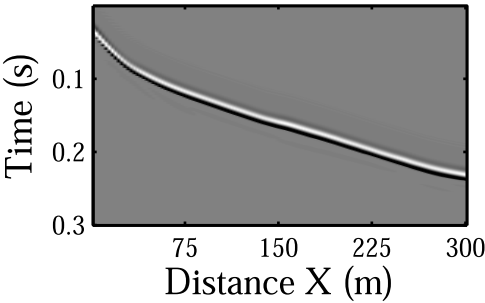

c)

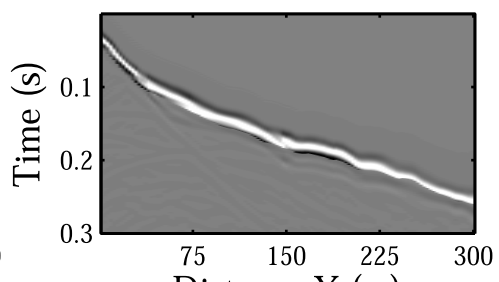

Distance X (m)

Fig. 21. First arrivals of CSGs \#1 with the tomograms inverted by (a) WT, (b) AE inversion (1-D), (c) envelope inversion, (d) AE inversion with 2-D latent space, and (e) FWI.

a)

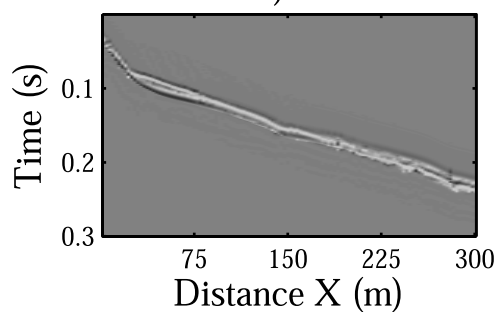

d)

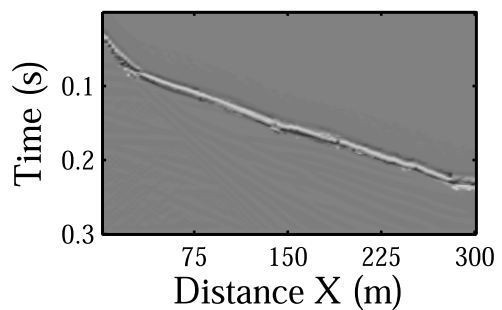

b)

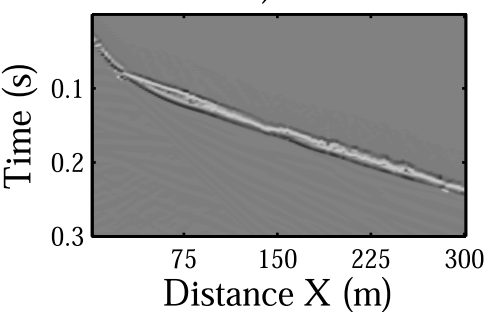

e)

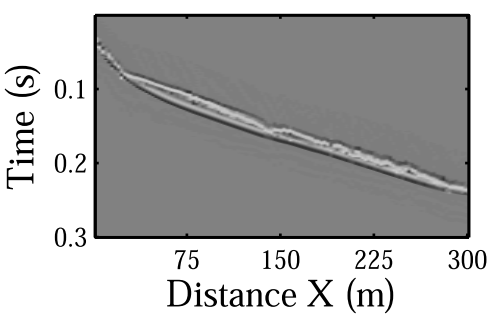

c)

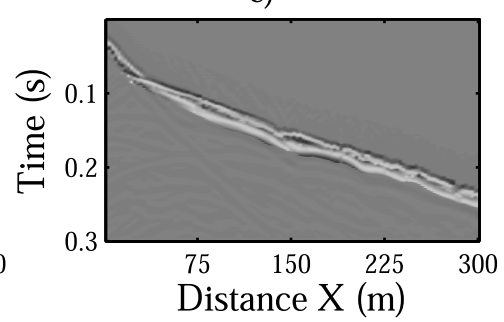

Distance X (m)

Fig. 22. First arrival residuals of CSGs \#1 associated with the raw data in Fig. 15(b) and the inverted data in Fig. 21(a)-(e). (a) Data residual with WT. (b) Data residual with AE Inv. (1-D). (c) Data residual with envelope Inv. (d) Data residual with AE Inv. (2-D). (e) Data residual with FWI.

true model in terms of the $L^{2}$ model residuals above the depth $17 \mathrm{~m}$. We calculate the model misfits above this depth because the refractional signals are often used for near-surface imaging. Since this AE inversion is supposed to include both the phase and the amplitude information through encoding, it performs better than WT even though its data misfit curve, i.e., the 2-D AE inversion, is a little inferior. Similar to FWI, the envelope inversion is still susceptible to the cycle-skipping problem although its input signal spectra are wide with some ultralow frequencies, which is also the reason why its inverted model is smoother than others. It is also worthy of attention that increasing the dimensions of the latent space can be beneficial for both the data compressing and the inversion. In the data domain, a higher dimensional latent space largely improves the quality of the decoded signals by looking over Figs. 7(a)-(b) and 8(a) because more degrees of freedom are
TABLE II

STATISTICAL FEATURES OF THE ENCODED LATENT SPace Values: Synthetic Data

\begin{tabular}{|c|c|c|c|}
\hline \multirow{2}{*}{ Encoded Properties } & \multirow{2}{*}{ 1D Latent Space } & \multicolumn{2}{|c|}{ 2D Latent Space } \\
\cline { 3 - 4 } & & First Dimension & Second Dimension \\
\hline Mean Value & 8.0676 & 3.3467 & 3.4168 \\
\hline Variance & 1.1699 & 0.8732 & 0.0515 \\
\hline
\end{tabular}

assigned to capture the envelope features (see Table II). As for the inverted tomograms [see Fig. 10(b) and (d)] by the two AE inversions, the interface between the two layers in Fig. 10(d) is a little, if not much, more accurate in-depth than that in Fig. 10(b), in spite that the interface shape in Fig. 10(b) shows stronger oscillations. Therefore, both the AE inversions (1-D and 2-D) can produce more robust and convincible results 
a)

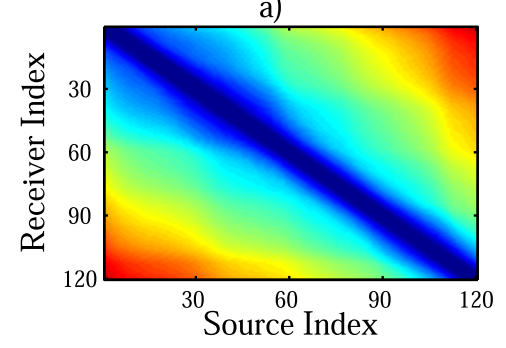

d)

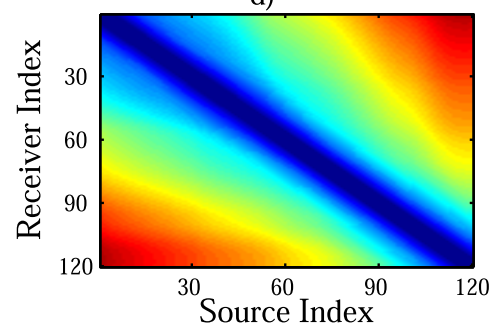

b)

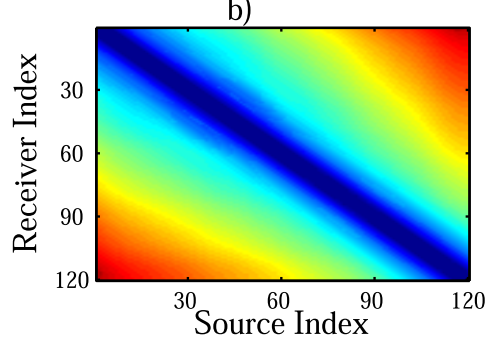

e)

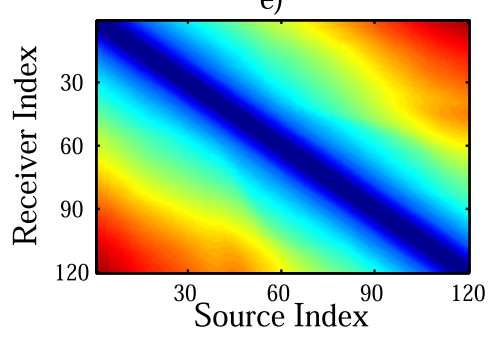

c)

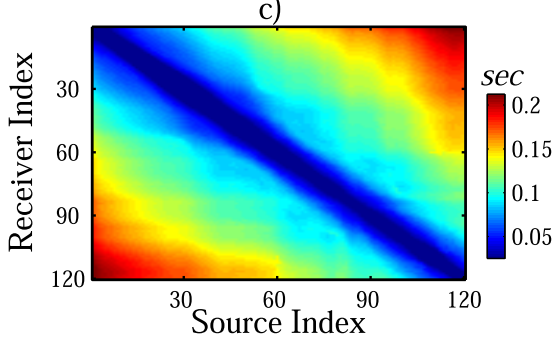

f)

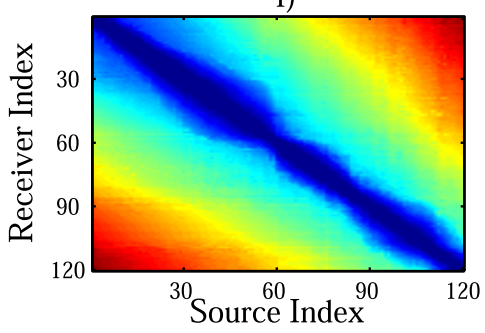

Fig. 23. First-arrival travel-time matrices with (a) WT, (b) AE inversion (1-D), (c) envelope inversion, (d) AE inversion (2-D), (e) FWI, and (f) picking. (a) First breaks with WT inversion. (b) First breaks with AE inversion (1-D). (c) First breaks with envelope inversion. (d) First breaks with AE inversion (2-D). (e) First breaks with FWI. (f) Picked first breaks.

by referring to Fig. 13(b). In practice, it is recommended that the AE inversions with both 1-D and 2-D latent spaces can be simultaneously conducted for promising results.

\section{B. Field Test on Aqaba Data}

A field data set collected near Aqaba, Jordan, to the north of the King Abdullah University of Science and Technology (KAUST), is shown in Fig. 14(a) [31]. The white line segment represents the 300-m-long survey line, where the ground elevation gradually increases uphill from west to east (see an elevation color bar shown on the right). The data are recorded by 120 receivers spaced at an interval of $2.5 \mathrm{~m}$, and they are triggered by 120 sources at every receiver's location. A vibrating source with a wide-frequency band is excited at every source location to conduct the experiment for all the CSGs. A typical CSG \#1 is shown in Fig. 14(b). The recording time for every CSG is $0.4 \mathrm{~s}$ with a sampling interval of $1 \mathrm{~ms}$, and the data in the last $0.1 \mathrm{~s}$ are muted as all the refractions arrive before $0.3 \mathrm{~s}$. For the convenience of computation, the raw CSGs are then downsampled by eight times by linear interpolation with the new temporal interval of $0.125 \mathrm{~ms}$. Therefore, we have $M=8 \times 0.3 \mathrm{~s} \div 1 \mathrm{~ms}=2400$, which is also the value of Dim1. For the horizontal and the vertical directions, we set the spatial grid size $d x=d z=0.625 \mathrm{~m}$.

Before the inversion, the raw CSGs are shifted and then simply processed by a median filter. The goal of the shifting operation is for the convenience of inversion because some first arrivals of the raw zero-offset traces are obscure, and this may cause frequency leaking during data processing. It can also make it difficult to match the very near-offset traces. Therefore, we shift all the CSGs forward in time by $20 \mathrm{~ms}$. All the first arrivals are windowed and extracted with their later arrivals muted by a tapered decaying window. Fig. 15(a) and (b) shows the processed CSG \#1 and its muted first arrivals to be inverted. Fig. 16(a) and 16(b), respectively,

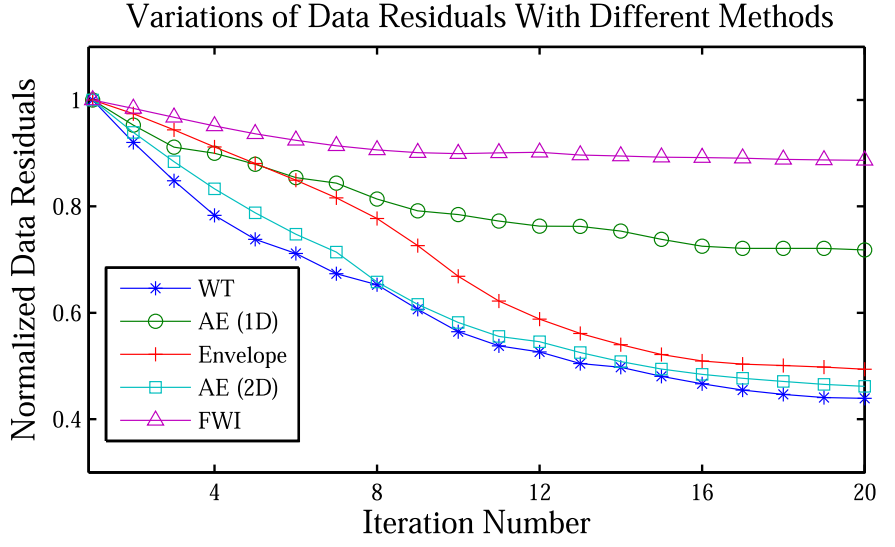

Fig. 24. Comparisons of data residuals with different methods.

shows the envelope presented in Fig. 15(a) and its decoded envelope by an autoencoding network.

In this land data case, we still set up two three-layered networks as the encoding and the decoding parts for training the input envelopes. Here, we set Dim1 $=2400, \operatorname{Dim} 2=800$, and $\operatorname{Dim} 3=150$. Therefore, the dimensions of the three encoding weighting matrices $W_{1}, W_{2}$, and $W_{3}$ are $2400 \times$ $800,800 \times 150$, and $150 \times 1$, respectively, in the forward direction. Accordingly, sizes of the three decoding matrices $W_{1}^{\prime}, W_{2}^{\prime}$, and $W_{3}^{\prime}$ are $800 \times 2400,150 \times 800$, and $1 \times 150$. All the encoded values of the first arriving envelopes (see Fig. 16(a) as an example), representing the scalars in the latent space, are presented in Fig. 16(c). Here, the learning rate is still set up as 0.001, and the iteration number is set as 80. Similarly, the dimension of the latent space can be set to two by keeping other preset parameters unchanged. Fig. 17(a) presents the decoded envelope of CSG \#1, which is much better than that shown in Fig. 16(b) compared with the standard envelope in Fig. 16(a). Fig. 17(b) and (c) presents 
a)

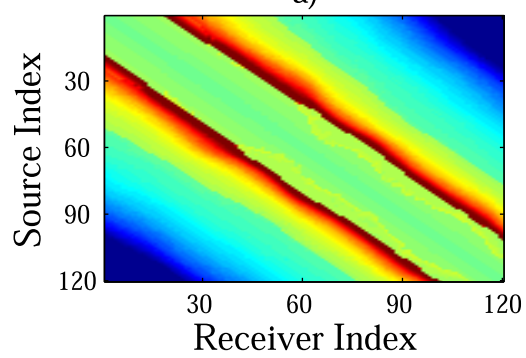

b)

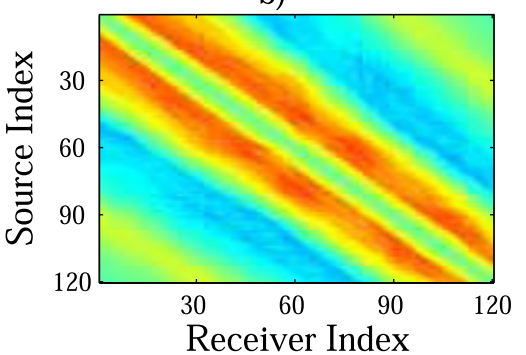

c)

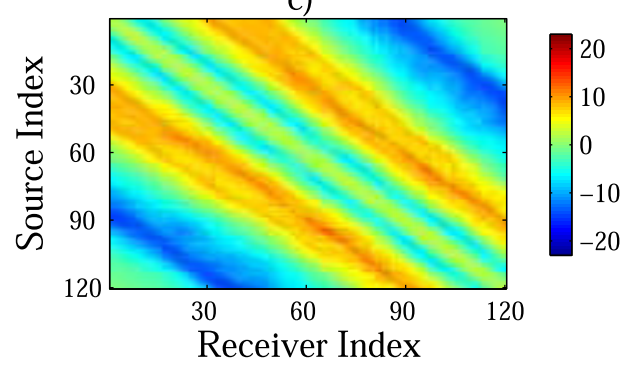

Fig. 25. (a) 1-D latent-space values for the AE inverted data and (b) first- and (c) second-dimensional latent-space values for the AE inverted data with 2-D latent space.

TABLE III

Statistical FEATURES OF THE ENCODED LATENT Space Values: AQABa Data

\begin{tabular}{|c|c|c|c|}
\hline \multirow{2}{*}{ Encoded Properties } & \multirow{2}{*}{ 1D Latent Space } & \multicolumn{2}{|c|}{ 2D Latent Space } \\
\cline { 3 - 4 } & & First Dimension & Second Dimension \\
\hline Mean Value & -3.0906 & 0.4376 & 0.4430 \\
\hline Variance & 9.9360 & 7.0920 & 7.5650 \\
\hline
\end{tabular}

the two encoded latent-space matrices for this field data set. Table III presents the statistical features of the encoded scalars and vectors of all the refractions in the Aqaba data set. Similar to the autoencoders in the synthetic case, both the absolute mean values and the variances' decay with the increment of latent space dimensions. It implies that there can be an abrupt or irregular variation of the 1-D latent scalar caused by perturbing the input waveforms, whereas such variation becomes relatively mild and robust in the 2-D case.

Five algorithms, i.e., the WT, the proposed AE inversions with both 1-D and 2-D latent spaces, the envelope inversion, and FWI are, respectively, applied to rebuild the subsurface structures for comparisons with the initial model presented in Fig. 18. After 20 iterations, the inverted models are shown in Fig. 19(a)-(e), and the first four inverted tomograms, especially the first and the fourth tomograms in Fig. 19(a) and (d), imply the possible location of Aqaba fault location at $X=125 \mathrm{~m}$ and $Z=20 \mathrm{~m}$, as indicated by the red curves in Fig. 14(a). The result is also consistent with the travel-time tomogram by Hanafy et al. [31]. Five gradient images of the first iteration are also presented in Fig. 20(a)-(e), which suggests that the skeletonized autoencoding inversion can reconstruct the essential geological features near the fault.

Two sanity tests are also conducted to validate the $\mathrm{AE}$ inverted results. First, five groups of first arrivals extracted from the forward modeled synthetic data based on the inverted tomograms are presented in Fig. 21(a)-(e). Their data differences from the raw CSG \#1 [see Fig. 15(b)] are presented in Fig. 22(a)-(e), and all the subfigures are normalized trace by trace. Second, the first-arrival travel-time matrices associated with the five inverted tomograms and the true model are presented in Fig. 23(a)-(f). As for the very near-offset first breaks, Fig. 23(a) is closer to the true first-arrival travel-time matrix presented in Fig. 23(f) although the quality of Fig. 23(d) is not inferior either. It means that WT is able to reconstruct the shallow parts of the subsurface structures in a more highly resolved sense. However, at a larger scale, Fig. 23(d) and (a) are more similar to Fig. 23(f) than Fig. 23(b), (c), and (e). Moreover, Fig. 23(d) presents a better prediction to the first arriving CSG \#1 than the travel times computed from the other four tomograms. All the data misfit curves with the five algorithms are presented in Fig. 24 as another quantitative reference. Here, the FWI misfit curve suggests that it is almost not able to update the starting model possible because of nonacoustic signals or unpredicted noise in the land data. Nevertheless, the envelope inversion performs better than FWI because low-frequency waveforms are particularly inverted, and they are less sensitive to the noise. The AE inversion with 1-D latent space cannot successfully produce the calculated data to be encoded for approximating the "observed" data in Fig. 16(c), and it may stop updating the velocity models after only a few iterations. The possible reason is that 1-D latent space is not adequate to express the first arrivals, especially in real applications with complex subsurface structures. Hence, it can cause nearly discontinuous encoding results, as shown in Fig. 16(c), especially when there are some jumps in neighbor traces. However, the situation becomes better in the 2-D case, whose tomogram is similar to that inverted by WT.

Unlike WT, which focuses on inverting the first breaks, or the envelope inversion, which recovers the envelope with a low-frequency band, the proposed AE inversion pays more attention to the skeletonized inner features of the first arriving signals. These vectors in the latent space are not referred to as specific physical quantities, but rather as certain numerical weights to modulate the quasi-envelope inversion shown in (15) and (26). As a comparison, the relationship of this "quasi-envelope" inversion and the envelope inversion is analogous to that between WT and the first arrival waveform inversion. The former methods invert the data based on its features, while the latter ones conduct inversions using waveforms. The theoretical similarities between the proposed $\mathrm{AE}$ inversion and WT are that both the implicit function theory and the connective function techniques are used. Fig. 25(a)-(c) presents the encoding results of two predicted data sets until the convergence of inversion as comparisons to Figs. 16(c) and 17(b) and (c). It once again verifies that an increase of the latent-space dimension can make a robust promise of the inversion quality in the data domain. 


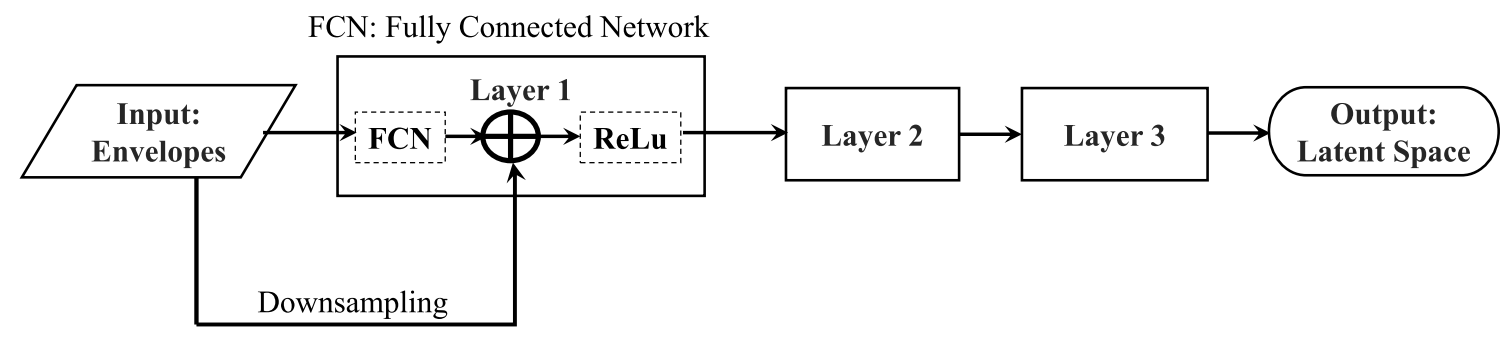

Fig. 26. Updated autoencoder with Resnet structure embedded in the encoding subnetwork.

\section{CONCLUSION AND Discussion}

We extended the wave-equation inversion of skeletonized data using a more state-of-the-art technique that minimizes the misfit function measuring the refraction differences. Applying an autoencoder to the windowed seismic data enables us to skeletonize them in the autoencoder's latent space, where the $\mathrm{AE}$ largely reduces the dimensionality of the input data to mitigate convergence problems with local minima. This compression allows for the computation of envelope derivatives with respect to the perturbation in the encoded latent space. The essential assumption meant by (7) and (20) is that the velocity perturbation is implicitly connected to the perturbation of the encoded values. Numerical results with both the synthetic and the field data sets validate the effectiveness of this skeletonized inversion with an autoencoder. It can accurately estimate the background velocity model with lowto-intermediate wavenumbers.

We also compared the $\mathrm{AE}$ inverted tomograms to those computed by three relevant techniques, WT, the envelope inversion, and FWI. FWI, with its misfit function highly nonlinear with respect to velocity perturbations, can be easily trapped into local minima especially when applying to real data. To alleviate this problem, WT and the envelope inversion were proposed in turn to effectively invert for more reliable velocity models. WT is usually robust and can produce an accurate background velocity model for inversion. However, it only uses the first breaks, which is usually time-consuming by manual picking or less reliable. Moreover, in the theory of WT, it is assumed that the first arrivals of the calculated and the observed data are identical in terms of their wavelets, and their only differences lie in the first breaks. Therefore, waveforms are not utilized in WT as in the skeletonized AE inversion, which unearths the information in the compressed first (or early) arrivals. However, it is not necessary to conduct careful and precise picking for $\mathrm{AE}$ inversion because the envelope encoding is automatically computed. The envelope inversion may bring some ultralow frequency information in the inversion because of the properties of the Hilbert transform. However, the encoder and decoder networks together try to extract the crucial components hidden in the first arriving waveforms. The skeletonization operation sheds light on the misfit functional that tends to reduce the differences in the observed and the simulated skeletal data but not the entire envelopes, hence making its convergence easier.

This article has illustrated that any type of seismological data sets can be represented in fewer dimensions through the autoencoder, thus transferring complexity into simplicity. In addition, if the encoded late-space variables are continuous with respect to the perturbation of the recorded data, then autoencoders may show great promise for a wide range of geophysical applications although we only conduct pure seismic inversions in this study. For regular encoding and decoding processes, the training actually behaves robust, and the gradients do not explode or vanish. To guarantee data consistency, the Resnet network is generally adopted with typical embedded skip-connections structures. Fig. 26 presents an updated autoencoder with Resnet in the encoding process, and it is also tested on the synthetic data set in the numerical section. Comparisons of training parameters using the regular encoder and the Resnet encoder are presented in Fig. 27. Both encoders show good convergence rates [see Fig. 27(a)], and the regular one can continuously update the weights of the first layer (Layer 1) with reasonable variances [see Fig. 27(b)], which means that the regular encoder without skip-connections will not stop optimizing the layers even far from the output as the iteration proceeds. In practice, it can be explained by the fact that the encoding network is not deep with only three layers, which is adequate for compressing regular refractions. Therefore, we have selected the empirical regular three-layered encoder and decoder as tools in this study, and this strategy is also for the purpose of saving computational time. Moreover, a higher dimensional latent space may bring more degrees of freedom to the data decoding and higher quality to the inversion. Therefore, depending on the complexity and the noise of the input data, it is necessary to choose a proper latent-space dimension. To the best of our knowledge, the pros and cons to weigh latent space variables differently in the misfit function are still unknown because they are both trained by such a straightforward encoder in this study but not by attention networks that are able to assign various meanings to the output terms. To optimize their weights in the misfit function, specifying the physical meanings of the encoded values, such as $z_{1}$ and $z_{2}$, is a necessary step for our future work. To a certain degree, these two variables denote some principle values of the first arriving wavelets extracted by the trained autoencoder. At this stage, it is uncertain if the two parameters are related to the amplitude and the phase information.

It is also worthwhile to point out that other machine learning methods, such as principle component analysis or convolutional neural networks, can be used to compress the data so that the implicit function theorem can be employed to 
a)

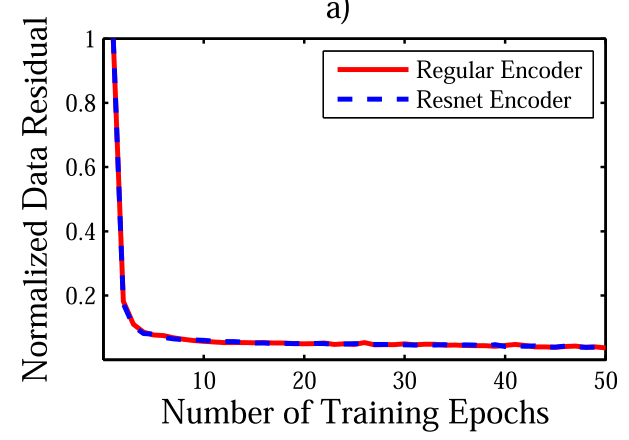

b)

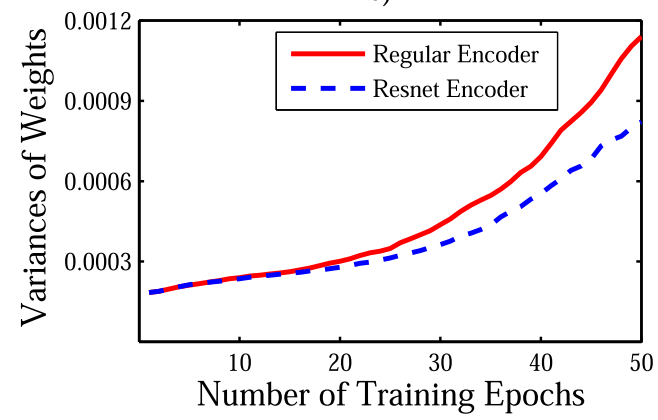

Fig. 27. Comparisons of the regular encoder and the Resnet encoder for their (a) convergence rates and (b) variances of their updated weights in the first layer for every training epoch.

invert the lower dimensional data. As a matter of fact, we have tested the $\mathrm{CNN}$ autoencoding (CAE) algorithm on the synthetic and the field data in this study, but the fully connectedlayer-based autoencoder is able to perform as well as, if not better than CAE. Both algorithms can bring the loss function down to $3 \%$, but $\mathrm{CAE}$ spends much more time in training and inversion (perhaps ten times more). We, thus, used the latter one for data processing and inversion. If complex later arriving waveforms are considered for inversion, then CAE can be a powerful tool for picking up local structures of the data. Another technique deserving attention is the automatic differentiation technique for implementing FWI [32], and it has the potential to replace the implicit function theorem. Automatic differentiation is a natural combination of neural network and FWI. In theory, data residuals in the latent space can be directly used to calculate the derivative of the misfit functional with respect to the velocity perturbations based on this technique. It is also one of our ongoing research topics. There are two significant topics to be explored in the future. The first one is how to reconstruct more complicated and noisy waveforms using autoencoders, which should consist of more layers and a multidimensional latent space. The second question to answer is how to produce a reasonable physical/ mathematical interpretation of the encoded values in the latent space.

\section{ACKNOWLEDGMENT}

The authors are very grateful to the anonymous reviewers for their precious comments and suggestions.

\section{REFERENCES}

[1] A. Tarantola, "Inversion of seismic reflection data in the acoustic approximation," Geophysics, vol. 49, no. 8, pp. 1259-1266, Aug. 1984.

[2] E. Baysal, D. D. Kosloff, and J. W. Sherwood, "Reverse time migration," Geophysics, vol. 48, no. 11, pp. 1514-1524, 1983.

[3] Z. Feng and G. T. Schuster, "Elastic least-squares reverse time migration," Geophysics, vol. 82, no. 2, pp. S143-S157, Mar. 2017.

[4] J. Virieux and S. Operto, "An overview of full-waveform inversion in exploration geophysics," Geophysics, vol. 74, no. 6, pp. WCC1-WCC26, Nov. 2009.

[5] P. C. Sava and S. Fomel, "Angle-domain common-image gathers by wavefield continuation methods," Geophysics, vol. 68, no. 3, pp. 1065-1074, 2003.

[6] C. Bunks, F. M. Saleck, S. Zaleski, and G. Chavent, "Multiscale seismic waveform inversion," Geophysics, vol. 60, no. 5, pp. 1457-1473, Sep. 1995
[7] L. Sirgue and R. G. Pratt, "Efficient waveform inversion and imaging: A strategy for selecting temporal frequencies," Geophysics, vol. 69, no. 1, pp. 231-248, Jan. 2004.

[8] B. Biondi and A. Almomin, "Tomographic full waveform inversion (TFWI) by combining full waveform inversion with wave-equation migration velocity anaylisis," in Proc. SEG Tech. Program Expanded Abstr., 2012, pp. 1-5.

[9] M. Warner and L. Guasch, "Adaptive waveform inversion: Theory," Geophysics, vol. 81, no. 6, pp. R429-R445, Nov. 2016.

[10] N. V. da Silva and G. Yao, "Wavefield reconstruction inversion with a multiplicative cost function," Inverse Problems, vol. 34, no. 1, Jan. 2018 , Art. no. 015004.

[11] R.-S. Wu and G. Chen, "Multiscale seismic-envelope inversion for salt structures using a new direct-envelope Fréchet derivative," in Proc. SEG Tech. Program Expanded Abstr., 2017, pp. 1523-1527.

[12] J. Kwon, H. Jin, H. Calandra, and C. Shin, "Interrelation between laplace constants and the gradient distortion effect in laplace-domain waveform inversion," Geophysics, vol. 82, no. 2, pp. R31-R47, Mar. 2017.

[13] G. Yao, N. V. da Silva, M. Warner, D. Wu, and C. Yang, "Tackling cycle skipping in full-waveform inversion with intermediate data," Geophysics, vol. 84, no. 3, pp. R411-R427, May 2019.

[14] Y. Chen, Z. Feng, L. Fu, A. AlTheyab, S. Feng, and G. Schuster, "Multiscale reflection phase inversion with migration deconvolution," Geophysics, vol. 85, no. 1, pp. R55-R73, Jan. 2020.

[15] Y. Sun and G. T. Schuster, "Time-domain phase inversion," in Proc. SEG Tech. Program Expanded Abstr., 1993, pp. 684-687.

[16] L. Fu, B. Guo, and G. T. Schuster, "Multiscale phase inversion of seismic data," Geophysics, vol. 83, no. 2, pp. R159-R171, Mar. 2018.

[17] Y. Luo, Y. Ma, Y. Wu, H. Liu, and L. Cao, "Full-traveltime inversionfulltraveltime inversion," Geophysics, vol. 81, no. 5, pp. R261-R274, 2016.

[18] Y. Luo and G. Schuster, "Wave-equation traveltime inversion," Geophysics, vol. 56, no. 5, pp. 645-653, 1991.

[19] Z. Liu and J. Zhang, "Joint traveltime, waveform, and waveform envelope inversion for near-surface imaging," Geophysics, vol. 82, no. 4, pp. R235-R244, Jul. 2017.

[20] G. Dutta, "Skeletonized wave-equation inversion for $\mathrm{Q}_{s}$," in Proc. SEG Tech. Program Expanded Abstr., 2016, pp. 3618-3623.

[21] J. Li, G. Dutta, and G. Schuster, "Wave-equation $\mathrm{Q}_{s}$ inversion of skeletonized surface waves," Geophys. J. Int., vol. 209, no. 2, pp. 979-991, May 2017.

[22] K. Lu, J. Li, B. Guo, L. Fu, and G. Schuster, "Tutorial for wave-equation inversion of skeletonized data," Interpretation, vol. 5, no. 3, pp. SO1SO10, Aug. 2017.

[23] Y. Chen and G. T. Schuster, "Seismic inversion by newtonian machine learning," 2019, arXiv:1904.10936. [Online]. Available: http://arxiv.org/abs/1904.10936

[24] A. Boesen Lindbo Larsen, S. Kaae Sønderby, H. Larochelle, and O. Winther, "Autoencoding beyond pixels using a learned similarity metric," 2015, arXiv:1512.09300. [Online]. Available: http://arxiv.org/abs/1512.09300

[25] A. P. Valentine and J. Trampert, "Data space reduction, quality assessment and searching of seismograms: Autoencoder networks for waveform data," Geophys. J. Int., vol. 189, no. 2, pp. 1183-1202, May 2012.

[26] Z. Camlica, H. R. Tizhoosh, and F. Khalvati, "Autoencoding the retrieval relevance of medical images," in Proc. Int. Conf. Image Process. Theory, Tools Appl. (IPTA), Nov. 2015, pp. 550-555. 
[27] J. Rao, M. Ratassepp, and Z. Fan, "Guided wave tomography based on full waveform inversion," IEEE Trans. Ultrason., Ferroelectr., Freq. Control, vol. 63, no. 5, pp. 737-745, May 2016.

[28] Z. Gao, Z. Pan, and J. Gao, "Multimutation differential evolution algorithm and its application to seismic inversion," IEEE Trans. Geosci. Remote Sens., vol. 54, no. 6, pp. 3626-3636, Jun. 2016.

[29] A. R. Levander, "Fourth-order finite-difference $P-S V$ seismograms," Geophysics, vol. 53, no. 11, pp. 1425-1437, 1988.

[30] J. Nocedal and S. Wright, Numerical Optimization. New York, NY, USA: Springer-Verlag, 2006.

[31] S. M. Hanafy, S. Jonsson, and Y. Klinger, "Imaging normal faults in alluvial fans using geophysical techniques: Field example from the coast of gulf of Aqaba, Saudi Arabia," in Proc. SEG Tech. Program Expanded Abstr., Aug. 2014, pp. 4670-4674.

[32] D. Cao and W. Liao, "A computational method for full waveform inversion of crosswell seismic data using automatic differentiation," Comput. Phys. Commun., vol. 188, pp. 47-58, Mar. 2015.

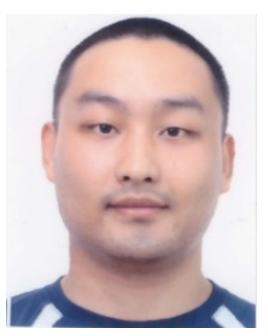

Han Yu received the Ph.D. degree in mathematics under the supervision of Craig C. Douglas from the University of Wyoming, Laramie, WY, USA, in 2011.

He was a Post-Doctoral Fellow with the Division of Physical Science and Engineering, King Abdullah University of Science and Technology (KAUST), Thuwal, Saudi Arabia, from 2012 to 2014. He was a semiprofessional swimmer in high school. He is also an Associate Professor with the School of Computer Science, Nanjing University of Posts and Telecommunications, Nanjing, China, and an Algorithm Consultant with Suzhou Keda Technology Company Ltd., Suzhou, China. His research interests include waveform inversion, numerical analysis, machine learning, and information security.

Dr. Yu is also a member of The Society of Exploration Geophysicists (SEG), the IEEE Computer Society (IEEE CS), China Computer Federation (CCF), and China Society for Industrial and Applied Mathematics (CSIAM).

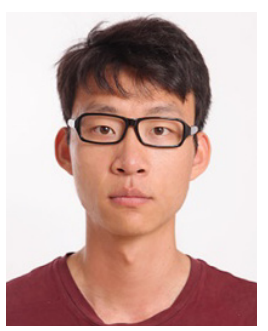

Yuqing Chen received the bachelor's degree in geophysics from the China University of Petroleum, Qingdao, China, in 2012, the master's degree in geophysics from the China University of Petroleum, Beijing, China, in 2015, and the Ph.D. degree in geophysics from the King Abdullah University of Science and Technology (KAUST), Thuwal, Saudi Arabia, in 2019

$\mathrm{He}$ is also a Post-Doctoral Fellow in the Deep Earth Imaging Future Science Platform, CSIRO, Canberra, ACT, Australia. His research interests include seismic inversion and machine learning.

Dr. Chen is also a member of The Society of Exploration Geophysicists (SEG), The European Association of Geoscientists and Engineers (EAGE), and American Geophysical Union (AGU).

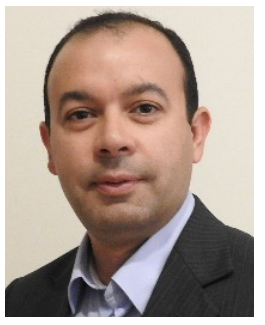

Sherif M. Hanafy received the B.Sc. and M.Sc. degrees in geophysics from Cairo University, Giza, Egypt, in 1993 and 1996, respectively, and the Ph.D. degree in geophysics from Kiel University, Kiel, Germany, in 2002.

$\mathrm{He}$ has more than 25 years of experience in academia and cooperation with the industry. He was a Senior Research Scientist with the King Abdullah University of Science and Technology (KAUST), Thuwal, Saudi Arabia, from 2009 to 2018, and an Assistant Professor with Cairo University from 2002 to 2009. He is also an Associate Professor of geophysics with the King Fahd University of Petroleum and Minerals, Dhahran, Saudi Arabia.

Dr. Hanafy received a DAAD scholarship to complete his Ph.D. degree in 1999. He received two scholarships to pursue his post-doctoral studies at The University of Utah, Salt Lake City, UT, USA, from 2004 to 2009.

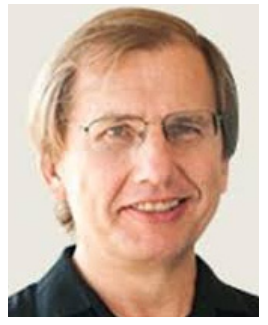

Gerard T. Schuster received the Ph.D. degree from Columbia University, New York, NY, USA, in 1984.

He was a Post-Doctoral Fellow with Columbia University from 1984 to 1985 . In fall 1985 , he began his duties as an Assistant Professor of geophysics at The University of Utah, Salt Lake City, UT, USA, where he was an Associate Professor from 1991 to 1996 and a Full Professor from 1997 to 2009. In 2009, he moved to the King Abdullah University of Science and Technology (KAUST), Thuwal, Saudi Arabia, as a Professor of Geophysics. His interests are in seismic imaging, interferometry, and machine learning and their applications to near-surface engineering, oil + mineral exploration, and paleoseismology. 